\title{
THE SUP-NORM VS. THE NORM OF THE COEFFICIENTS: EQUIVALENCE CONSTANTS FOR HOMOGENEOUS POLYNOMIALS
}

\author{
DANIEL GALICER, MARTÍN MANSILLA, AND SANTIAGO MURO
}

\begin{abstract}
Let $A_{p, r}^{m}(n)$ be the best constant that fulfills the following inequality: for every $m$ homogeneous polynomial $P(z)=\sum_{|\alpha|=m} a_{\alpha} z^{\alpha}$ in $n$ complex variables,$$
\left(\sum_{|\alpha|=m}\left|a_{\alpha}\right|^{r}\right)^{1 / r} \leq A_{p, r}^{m}(n) \sup _{z \in B_{\ell_{p}^{n}}^{n}}|P(z)| .
$$

For every degree $m$, and a wide range of values of $p, r \in[1, \infty]$ (including any $r$ in the case $p \in[1,2]$, and any $r$ and $p$ for the 2-homogeneous case), we give the correct asymptotic behavior of these constants as $n$ (the number of variables) tends to infinity. Remarkably, in many cases, extremal polynomials for these inequalities are not (as traditionally expected) found using classical random unimodular polynomials, and special combinatorial configurations of monomials are needed. Namely, we show that Steiner polynomials (i.e., $m$-homogeneous polynomials such that the multi-indices corresponding to the nonzero coefficients form partial Steiner systems), do the work for certain range of values of $p, r$.

As a byproduct, we present some applications of these estimates to the interpolation of tensor products of Banach spaces, to the study of (mixed) unconditionality in spaces of polynomials and to the multivariable von Neumann's inequality.
\end{abstract}

\section{INTRODUCTION}

As usual we denote $\ell_{p}^{n}$ for the Banach space of all $n$-tuples $z=\left(z_{1}, \ldots, z_{n}\right) \in \mathbb{C}^{n}$ endowed with the norm $\left\|\left(z_{1}, \ldots, z_{n}\right)\right\|_{p}=\left(\sum_{i=1}^{n}\left|z_{i}\right|^{p}\right)^{1 / p}$ if $1 \leq p<\infty$, and $\left\|\left(z_{1}, \ldots, z_{n}\right)\right\|_{\infty}=\max _{i=1, \ldots, n}\left|z_{i}\right|$ for $p=\infty$. The unit ball of $\ell_{p}^{n}$ is denoted by $B_{\ell_{p}^{n}}$. For $1 \leq p \leq \infty$ we write $p^{\prime}$ for its conjugate exponent (i.e., $\frac{1}{p}+\frac{1}{p^{\prime}}=1$ ).

2010 Mathematics Subject Classification. 46G25,15A60,47H60,11C08,15A69,47A30.

Key words and phrases. Hardy-Littlewood inequalities, unimodular polynomials, unconditionality in spaces of polynomials, multivariable von Neumann's inequality.

This work was partially supported by projects CONICET PIP 11220130100329CO, ANPCyT PICT 20152224, ANPCyT PICT 2015-2299, ANPCyT PICT 2015 - 3085, UBACyT 20020130100474BA, UBACyT 20020130300052BA, UBACyT 20020130300057BA. The second author was supported by a CONICET doctoral fellowship. 
An $m$-homogeneous polynomial in $n$ variables is a function $P: \mathbb{C}^{n} \rightarrow \mathbb{C}$ of the form

$$
P\left(z_{1}, \ldots, z_{n}\right)=\sum_{\alpha \in \Lambda(m, n)} a_{\alpha} z^{\alpha}
$$

where $\Lambda(m, n):=\left\{\alpha \in \mathbb{N}_{0}^{n}:|\alpha|:=\alpha_{1}+\cdots+\alpha_{n}=m\right\}, z^{\alpha}:=z_{1}^{\alpha_{1}} \cdots z_{n}^{\alpha_{n}}$ and $a_{\alpha} \in \mathbb{C}$.

Another way of writing a polynomial $P$ is as follows:

$$
P\left(z_{1}, \ldots, z_{n}\right)=\sum_{\mathbf{j} \in \mathcal{J}(m, n)} c_{\mathbf{j}} z_{\mathbf{j}}
$$

where $\mathcal{J}(m, n):=\left\{\mathbf{j}=\left(j_{1}, \ldots, j_{k}\right): 1 \leq j_{1} \leq \ldots \leq j_{k} \leq n\right\}, z_{\mathbf{j}}:=z_{j_{1}} \cdots z_{j_{k}}$ and $c_{\mathbf{j}} \in \mathbb{C}$. Note that $c_{\mathbf{j}}=a_{\alpha}$ with $\mathbf{j}=\left(1, . \alpha_{1} ., 1, \ldots, n, \alpha_{m} ., n\right)$.

We refer to the elements $\left(z^{\alpha}\right)_{\alpha \in \Lambda(m, n)}$ (equivalently, $\left.\left(z_{\mathbf{j}}\right)_{\mathbf{j} \in \mathcal{J}(m, n)}\right)$ as the monomials.

For $1 \leq p \leq \infty$ we denote by $\mathcal{P}\left({ }^{m} \ell_{p}^{n}\right)$ the Banach space of all $m$-homogeneous polynomials in $n$ complex variables equipped with the uniform (or sup) norm

$$
\|P\|_{\mathcal{P}\left({ }^{m} \ell_{p}^{n}\right)}:=\sup _{z \in B_{\ell_{p}^{n}}}|P(z)|
$$

Given an $m$-homogeneous polynomial $P(z)=\sum_{\alpha \in \Lambda(m, n)} a_{\alpha} z^{\alpha}$ in $n$ variables we denote the $\ell_{r}$-norm of its coefficients by

$$
|P|_{r}:=\left(\sum_{\alpha \in \Lambda(m, n)}\left|c_{\alpha}\right|^{r}\right)^{1 / r} .
$$

Other norm, related to the coefficients is the so-called Bombieri $r$-norm defined in [BBEM90]:

$$
[P]_{r}:=\left(\sum_{\alpha \in \Lambda(m, n)}\left(\frac{\alpha !}{m !}\right)^{r-1}\left|c_{\alpha}\right|^{r}\right)^{1 / r} .
$$

The relation between the these coefficients-norms is given by the following inequalities (see [BBEM90]):

$$
(m !)^{\frac{1}{r}-1}|P|_{r} \leq[P]_{r} \leq|P|_{r}
$$

In many contexts, it is essential to relate the summability of the coefficients of a given homogeneous polynomial with the sup-norm on the unit ball of the ambient space. This type of comparison results have shown several applications to different problems in complex and harmonic analysis, number theory and even in modern physics. Among them we include the contributions to the study of: the asymptotic behavior of the Bohr radius [DFOC ${ }^{+} 11$, BPSS14, DF11] or Dirichlet-Bohr radius $\mathrm{CDG}^{+} 14$, Sidon constants and convergence of Dirichlet series BCQ06, BDS15, BH31, DFOC ${ }^{+}$11, dLB08, KQ01, Que95, monomial series expansions of holomorphic 
functions in infinitely many variables and multipliers of Dirichlet series [BDF+14, BDS15], classical inequalities in operator theory [Dix76, MT79, GMSP15] and lower bounds on the classical bias obtainable in multiplayer XOR games [Mon12].

Most of the applications mentioned above rely on the ingenious use of the celebrated BohnenblustHille inequality (see [BPSS14, $\left.\mathrm{DFOC}^{+} 11\right]$ ). This inequality is a generalization to higher degrees of Littlewood's classical 4/3-inequality [Lit30] (a forerunner of Grothendieck's inequality) and essentially bounds the $\ell_{\frac{2 m}{m+1}}$-norm of the coefficients of an $m$-homogeneous polynomial in terms of its uniform norm on the polydisk. More precisely,

Theorem 1.1 (Bohnenblust-Hille inequality). There is a constant $C_{m, \infty}>0$ (which depends on $m$ but not on $n$ ) such that, for every $m$-homogeneous polynomial $P$ in any number of complex variables $n$, we have:

$$
|P|_{\frac{2 m}{m+1}} \leq C_{m, \infty}\|P\|_{\mathcal{P}\left(m \ell_{\infty}^{n}\right)}
$$

Moreover, the $\ell_{\frac{2 m}{m+1}}$-norm of the coefficients on the left hand side is optimal: i.e., there is no similar inequality replacing this norm by other $\ell_{r}$-norm, for $r<\frac{2 m}{m+1}$, involving a constant independent of the number of variables.

There are a number of analogues/generalizations of this inequality (which maintain the philosophy that the constant involved is totally independent of the number of variables), and consist in replacing the sup-norm on the ball of $\ell_{\infty}^{n}$ on the right hand side of the Bohnenblust-Hille inequality by other $\ell_{p}^{n}$-uniform norms (obviously changing the summability condition on the left side). These generalizations, inspired by some classical inequalities for bilinear forms [HL34], are known today in the literature as Hardy-Littlewood inequalities for homogenous polynomials and they have been carefully studied during the last years [ABPSS13, DSP13, PP81]. Precisely,

Theorem 1.2 (Hardy-Littlewood type inequalities). There is a constant $C_{m, p}>0$ (only depending on $m$ and $p$ and independent of $n$ ) such that for every $m$-homogeneous polynomial in $n$-complex variables we have:

$$
\begin{aligned}
& |P|_{\frac{p}{p-m}} \leq C_{m, p}\|P\|_{\mathcal{P}\left(m \ell_{p}^{n}\right)} \quad \text { for } m \leq p \leq 2 m, \\
& |P|_{\frac{2 m p}{m p+p-2 m}} \leq C_{m, p}\|P\|_{\mathcal{P}\left(m \ell_{p}^{n}\right)} \quad \text { for } 2 m \leq p \text {. }
\end{aligned}
$$

Again the exponents $\frac{p}{p-m}$ and $\frac{2 m p}{m p+p-2 m}$ in the above inequalities are the best possible. Observe that, in the limit case $(p=\infty)$ we recover the classical Bohnenblust-Hille exponent $\frac{2 m}{m+1}$.

If we change any of the parameters involved on either or both sides of these Hardy-Littlewood inequalities, it is expected that the dependence on the number of variables becomes apparent. It is worth asking how this reliance is in terms of the summability of the coefficients, the uniform 
norm and the homogeneity degree considered.

Analogously, we can study a similar problem: the inequality that comes from exchanging the roles (sides of the inequality) between the norm of the coefficients and the uniform norm.

Problem 1.3. Let $A_{p, r}^{m}(n)$ and $B_{r, p}^{m}(n)$ be the smallest constants that fulfill the following inequalities: for every $m$-homogeneous polynomial $P$ in $n$ complex variables,

$$
\begin{aligned}
|P|_{r} & \leq A_{p, r}^{m}(n)\|P\|_{\mathcal{P}\left({ }^{m} \ell_{p}^{n}\right)}, \\
\|P\|_{\mathcal{P}\left({ }^{m} \ell_{p}^{n}\right)} & \leq B_{r, p}^{m}(n)|P|_{r} .
\end{aligned}
$$

How these constants behave in terms of the number of variables $n$ ? Which is their exact asymptotic growth?

Observe that by (11), the depence on $n$ of the constant that appears when comparing the sup-norm with the Bombieri norm is exactly the same as the constants related to Problem 1.3.

In the 80's, Goldberg [Gol87] settled a similar problem in the context of matrix theory: given an $n \times n$ matrix $A$, he was interested in finding the best equivalence constant $c(r, p, n)$ (or its asymptotic behavior as $n$ tends to infinity) which relates the $\ell_{r}$-norm of the coefficients with the operator norm of $A$ acting on $\ell_{p}^{n}$. Partial and sharp results of this problem (and also some variants of it) were given by Feng and Tonge in [Ton00, Fen03, FT07]. Observe that Problem 1.3 is essentially a polynomial version of Golberg's problem. Of course, this can also be settled for multilinear forms, whose constants turn out to have the same asymptotic growth. Indeed, note that using the notation of [DMP09], $A_{p, r}^{m}(n)=\left\|i d: \otimes_{\varepsilon_{s}}^{m, s} \ell_{p^{\prime}}^{n} \rightarrow \otimes_{\Delta_{r}}^{m, s} \ell_{r}^{n}\right\|$. In [DMP09, Proposition 3.1.] it is proved that

$$
\left\|i d: \otimes_{\varepsilon_{s}}^{m, s} \ell_{p^{\prime}}^{n} \rightarrow \otimes_{\Delta_{r}}^{m, s} \ell_{r}^{n}\right\| \sim\left\|i d: \otimes_{\varepsilon}^{m} \ell_{p^{\prime}}^{n} \rightarrow \otimes_{\Delta_{r}}^{m} \ell_{r}^{n}\right\|
$$

This implies that the asymptotic behaviour of the polynomial and the multilinear cases are the same. We should mention that some partial results for the multilinear problem were recently obtained by Araujo and Pellegrino [AP15] (see also [BMP10] for the case $p=2$ ).

For every degree $m$ and a wide range of values of $p, r \in[1, \infty]$, we give in Theorem 2.1 the correct asymptotic behavior of $A_{p, r}^{m}(n)$ as $n$ tends to infinity respectively. We also present in Proposition 2.4 the asymptotic growth of $B_{r, p}^{m}(n)$ for every $p, r \in[1, \infty]$. We also use these results to tackle two different problems: we present some applications of our estimates to the study of unconditionality in spaces of polynomials and the multivariable von Neumann's inequality. 
Definition 1.4. Let $\left(P_{i}\right)_{i \in \Lambda}$ be a Schauder basis of $\mathcal{P}\left({ }^{m} \mathbb{C}^{n}\right)$. For $1 \leq p, q \leq \infty$ and $n, m \in \mathbb{N}$ let $\chi_{p, q}\left(\left(P_{i}\right)_{i \in \Lambda}\right)$ be the best constant $C>0$ such that

$$
\left\|\sum_{i \in \Lambda} \theta_{i} c_{i} P_{i}\right\|_{\mathcal{P}\left(m \ell_{q}^{n}\right)} \leq C\left\|\sum_{i \in \Lambda} c_{i} P_{i}\right\|_{\mathcal{P}\left(m \ell_{p}^{n}\right)}
$$

for every $P=\sum_{i \in \Lambda} c_{i} P_{i} \in \mathcal{P}\left({ }^{m} \mathbb{C}^{n}\right)$ and every choice of complex numbers $\left(\theta_{i}\right)_{i \in \Lambda}$ of modulus one.

The $(p, q)$-mixed unconditional constant of $\mathcal{P}\left({ }^{m} \mathbb{C}^{n}\right)$ is defined as

$$
\chi_{p, q}\left(\mathcal{P}\left({ }^{m} \mathbb{C}^{n}\right)\right):=\inf \left\{\chi_{p, q}\left(\left(P_{i}\right)_{i \in \Lambda}\right):\left(P_{i}\right)_{i \in \Lambda} \text { basis for } \mathcal{P}\left({ }^{m} \mathbb{C}^{n}\right)\right\}
$$

This notion was introduced by Defant, Maestre and Prengel in [DMP09, Section 5].

In Section 3 we provide correct estimates of the asymptotic growth of the mixed- $(p, q)$ unconditional constant as $n$ tends to infinity. To achieve this we use some major results given in [DMP09, BDS15] about domains of monomial convergence, our bounds on Problem 1.3 and multilinear interpolation. Moreover, we give an analog of a result of Pisier and Schütt [Pis78, Sch78] in this context. Namely, in order to study the asymptotic behavior of the mixed unconditional constants of $\mathcal{P}\left({ }^{m} \mathbb{C}^{n}\right)$, it is enough to look at the monomials $\left(z_{\mathbf{j}}\right)_{\mathbf{j} \in \mathcal{J}(m, n)}$. More precisely, we prove in Theorem 3.1 that

$$
\chi_{p, q}\left(\mathcal{P}\left({ }^{m} \mathbb{C}^{n}\right)\right) \sim \chi_{p, q}\left(\left(z_{\mathbf{j}}\right)_{\mathbf{j} \in \mathcal{J}(m, n)}\right) .
$$

We feel this result is interesting in its own right.

Mantero and Tonge considered in [MT79] several versions of the multivariable von Neumann's inequality restricted to homogeneous polynomial on several commuting operators. Among them, they were interested in the asymptotic behavior of the best possible constant $c(n)=c_{m, p, q}(n)$ such that

$$
\left\|P\left(T_{1}, \ldots, T_{n}\right)\right\|_{\mathcal{L}(\mathcal{H})} \leq c(n)\|P\|_{\mathcal{P}\left({ }^{m} \ell_{q}^{n}\right)},
$$

for every $n$-tuple $T_{1}, \ldots, T_{n}$ of commuting operators on a Hilbert space $\mathcal{H}$ satisfying

$$
\sum_{i=1}^{n}\left\|T_{i}\right\|_{\mathcal{L}(\mathcal{H})}^{p} \leq 1
$$

and every $m$-homogeneous polynomial $P$ in $n$-complex variables. We use our estimates on HardyLittlewood type inequalities to obtain new upper bounds for the behavior of $c(n)$, and also address a related problem also treated in [MT79.

Before we present and prove our main results, we give some important comments. To show that our estimates are sharp it is essential to have polynomials with small norm with many nonzero coefficients. A widely used technique to find these extremal polynomials is given by 
the probabilistic method: i.e., considering an $m$-homogeneous polynomial whose coefficients are given by independent random variables and computing it expectation (pretending to be small). The systematic study of norms of random homogeneous polynomials arguably goes back to the classical Kahane-Salem-Zygmund theorem [Kah93, Chapter 6], which was found very useful in harmonic analysis. Recently, additional applications of random (homogeneous) polynomials were given to complex and functional analysis and to operator theory (see for example [MT80, Boa00, DDGM01, DGM03, DGM04, CD07, Bay12).

Bayart in Bay12] (see also [Boa00, DGM03, DGM04]) exhibited polynomials with unimodular coefficients and with small sup-norm on the unit ball of $\ell_{p}^{n}$. He showed that for each $1 \leq p \leq \infty$ there exists an $m$-homogeneous unimodular polynomial $P(z):=\sum_{\alpha \in \Lambda(m, n)} \varepsilon_{\alpha} z^{\alpha}$ (i.e., $\varepsilon_{\alpha}= \pm 1$ for every $\alpha$ ) in $n$ complex variables and a constant $K_{m, p}$ that depends exclusively on $m$ and $p$ such that

$$
\|P\|_{\mathcal{P}\left(m \ell_{p}^{n}\right)} \leq K_{m, p} \times \begin{cases}n^{1-\frac{1}{p}} & \text { if } 1 \leq p \leq 2 \\ n^{m\left(\frac{1}{2}-\frac{1}{p}\right)+\frac{1}{2}} & \text { if } 2 \leq p \leq \infty\end{cases}
$$

Observe that the number of non-zero coefficients is exactly the number of possible monomials, $\left(\begin{array}{c}n+m-1 \\ m\end{array}\right)$. These polynomials will be very useful: they will be extremal in many ranges of values of $p, r \in[1, \infty]$ for the first inequality of Problem 1.3. Unfortunately, for a large range of values of $p$ and $r$ these polynomials become useless and new extremal examples are needed. Therefore, it is important to relax the number of terms appearing in the polynomials, by allowing them to have some zero coefficients, in order to reduce quantitatively the value of the sup-norm. Obviously if one gets rid of many coefficients/monomials this helps considerably to lower the value of the norm but the important thing is to maintain an appropriate balance (having a sufficient number of non-zero coefficients but keeping the norm small).

We show that so-called Steiner polynomials, a special class of tetrahedral polynomials introduced by Dixon in [Dix76] and studied in [GMSP15] are accurate enough for our purposes. Beyond the results exhibited in this article, this shows once again that Steiner polynomials play an important role in the area. We believe that this motivates to study in depth these polynomials and its possible future applications.

We need some definitions to describe them. An $S_{p}(t, m, n)$ partial Steiner system is a collection of subsets of size $m$ of $\{1, \ldots, n\}$ such that every subset of $t$ elements is contained in at most one member of the collection of subsets of size $m$. An $m$-homogeneous polynomial $P$ of $n$ variables is a Steiner polynomial if there exists an $S_{p}(t, m, n)$ partial Steiner system $\mathcal{S}$ such that 
$P\left(z_{1}, \ldots, z_{n}\right)=\sum_{\mathbf{j} \in \mathcal{S}} c_{\mathbf{j}} z_{\mathbf{j}}$ and $c_{\mathbf{j}}= \pm 1$. Note that the monomials involved in this class have a particular combinatorial configuration.

The following result appears in [GMSP15, Theorem 2.5.].

Theorem 1.5. Let $m \geq 2$ and $\mathcal{S}$ be an $S_{p}(m-1, m, n)$ partial Steiner system. Then there exist signs $\left(c_{\mathbf{j}}\right)_{\mathbf{j} \in \mathcal{S}}$ and a constant $D_{m, p}>0$ independent of $n$ such that the $m$-homogeneous polynomial $P=\sum_{\mathbf{j} \in \mathcal{S}} c_{\mathbf{j}} z_{\mathbf{j}}$ satisfies

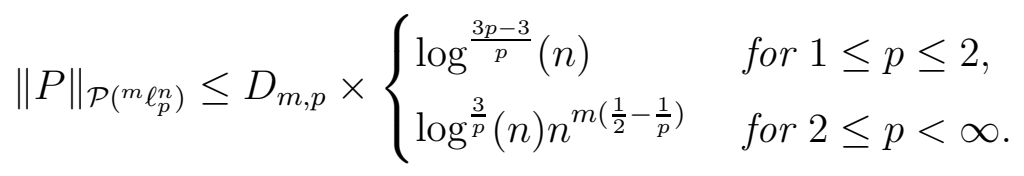

Moreover, the constant $D_{m, p}$ may be taken independent of $m$ for $p \neq 2$.

The last ingredient we need for the applications is the existence of nearly optimal partial Steiner systems, in the sense that they have many elements. This translates to many unimodular coefficients of the Steiner polynomials. It is well known that any partial Steiner system $S_{p}(m-$ $1, m, n)$ has cardinality less than or equal to $\frac{1}{m}\left(\begin{array}{c}n \\ m-1\end{array}\right)$.

Rödl [Röd85] in the eighties proved that there exist partial Steiner systems $S_{p}(m-1, m, n)$ of cardinality at least $(1-o(1)) \frac{1}{m}\left(\begin{array}{c}n \\ m-1\end{array}\right)$, where $o(1)$ tends to zero as $n$ goes to infinity. Taking partial Steiner systems of this cardinality in Theorem 1.5 we have the following.

Corollary 1.6. Let $m \geq 2$. Then there exists a $m$-homogeneous Steiner unimodular polynomial $P$ of $n$ complex variables with at least $C_{m} n^{m-1}$ unimodular coefficients satisfying the estimates in Theorem 1.5, where $C_{m}$ is a constant that depends only on $m$.

The article is organized as follows. In Section 2 we address Problem 1.3. Section 3 deals with the study of (mixed) unconditionality in spaces of polynomials. Finally, Section 4 presents some applications to several versions of the multivariable von Neumann's inequality.

\section{MAin RESUlts}

If $\left(a_{n}\right)_{n}$ and $\left(b_{n}\right)_{n}$ are two sequences of real numbers we will write $a_{n} \ll b_{n}$ if there exists a constant $C>0$ (independent of $n$ ) such that $a_{n} \leq C b_{n}$ for every $n$. We will write $a_{n} \sim b_{n}$ if $a_{n} \ll b_{n}$ and $b_{n} \ll a_{n}$. Recall that the number of $m$-homogeneous monomials in $n$ variables is $|\mathcal{J}(m, n)|=\left(\begin{array}{c}n+m-1 \\ m\end{array}\right) \sim n^{m}$.

For every $P \in \mathcal{P}\left({ }^{m} \mathbb{C}^{n}\right)$ there exists a unique symmetric $m$-linear form $T$ such that for every $x \in \mathbb{C}^{n}, P(x)=T(x, \stackrel{m}{.}, x)$ (see [Din99]). We will denote the $r$-th coefficients norm of $T$ by $|T|_{r}$, 
that is,

$$
|T|_{r}:=\left(\sum_{i \in \mathcal{M}(m, n)}\left|T\left(e_{i_{1}}, \ldots, e_{i_{m}}\right)\right|^{r}\right)^{\frac{1}{r}},
$$

where $\mathcal{M}(m, n)=\left\{\mathbf{i}=\left(i_{1}, \ldots, i_{m}\right): 1 \leq i_{l} \leq n, 1 \leq l \leq m\right\}$. It is well known that there exist constants $C_{l}=C_{l}(m)>0, l=1,2$, independent of $n$, such that for every $P \in \mathcal{P}\left({ }^{m} \mathbb{C}^{n}\right)$ and its associated symmetric $m$-linear form $T$ we have

$$
\begin{aligned}
|T|_{r} & \leq|P|_{r} \leq C_{1}|T|_{r} & & \text { for } 1 \leq r \leq \infty \\
C_{2}\|T\|_{\mathcal{L}\left({ }^{m} \ell_{p}^{n}\right)} & \leq\|P\|_{\mathcal{P}\left({ }^{m} \ell_{p}^{n}\right)} \leq\|T\|_{\mathcal{L}\left({ }^{m} \ell_{p}^{n}\right)} & & \text { for } 1 \leq p \leq \infty .
\end{aligned}
$$

We now state our main theorem.

Theorem 2.1. Let $A_{p, r}^{m}(n)$ be the smallest constant such that for every m-homogeneous polynomial $P$ in $n$ complex variables,

$$
|P|_{r} \leq A_{p, r}^{m}(n)\|P\|_{\mathcal{P}\left(m \ell_{p}^{n}\right)}
$$

Then,

$$
\left\{\begin{aligned}
A_{p, r}^{m}(n) & \sim 1 & & \text { for }(A):\left[\frac{1}{2} \leq \frac{1}{r} \leq \frac{m+1}{2 m}-\frac{1}{p}\right] \text { or }\left[\frac{1}{r} \leq \frac{1}{2} \wedge \frac{m}{p} \leq 1-\frac{1}{r}\right], \\
A_{p, r}^{m}(n) & \sim n^{\frac{m}{p}+\frac{1}{r}-1} & & \text { for }(B):\left[\frac{1}{2 m} \leq \frac{1}{p} \leq \frac{1}{m} \wedge-\frac{m}{p}+1 \leq \frac{1}{r}\right], \\
A_{p, r}^{m}(n) & \sim n^{m\left(\frac{1}{p}+\frac{1}{r}-\frac{1}{2}\right)-\frac{1}{2}} & & \text { for }(C):\left[\frac{m+1}{2 m} \leq \frac{1}{r} \wedge \frac{1}{p} \leq \frac{1}{2}\right] \text { or }\left[\frac{1}{2} \leq \frac{1}{r} \leq \frac{m+1}{2 m} \leq \frac{1}{p}+\frac{1}{r} \wedge \frac{1}{p} \leq \frac{1}{2}\right], \\
A_{p, r}^{m}(n) & \sim n^{\frac{m}{r}+\frac{1}{p}-1} & & \text { for }(D):\left[\frac{1}{2} \leq \frac{1}{p} \wedge 1-\frac{1}{p} \leq \frac{1}{r}\right], \\
A_{p, r}^{m}(n) & \ll n^{\frac{m-1}{r}} & & \text { for }(E):\left[\frac{1}{2} \leq \frac{1}{p} \leq 1-\frac{1}{r}\right], \\
A_{p, r}^{m}(n) & \sim n^{\frac{1}{r}} & & \text { for }(F):\left[\frac{m-1}{p} \leq 1-\frac{1}{r} \wedge \frac{1}{m} \leq \frac{1}{p} \leq \frac{1}{m-1}\right],
\end{aligned}\right.
$$

Moreover, the power of $n$ in $(E)$ cannot be improved.

The first figure represents the regions described in Theorem 2.1. For the blank region we do not know right order of $A_{p, r}^{m}(n)$ (see the comments after Remark 2.3 below). It is noteworthy that much of the work is to determine which are the regions to consider.

Also note that for $m=2$ we have a complete description of the asymptotics of $A_{p, r}^{2}(n)$. For $r \geq 2$ this can also be deduced as a consequence of [FT07, Theorems 1 and 2]. For $r<2$ the results are, up to our knowledge, new.

Proof. Let $P$ be an $m$-homogeneous polynomial in $n$ complex variables and $T$ its associated symmetric $m$-linear form. We will use several times the following inequalities, which are a simple 


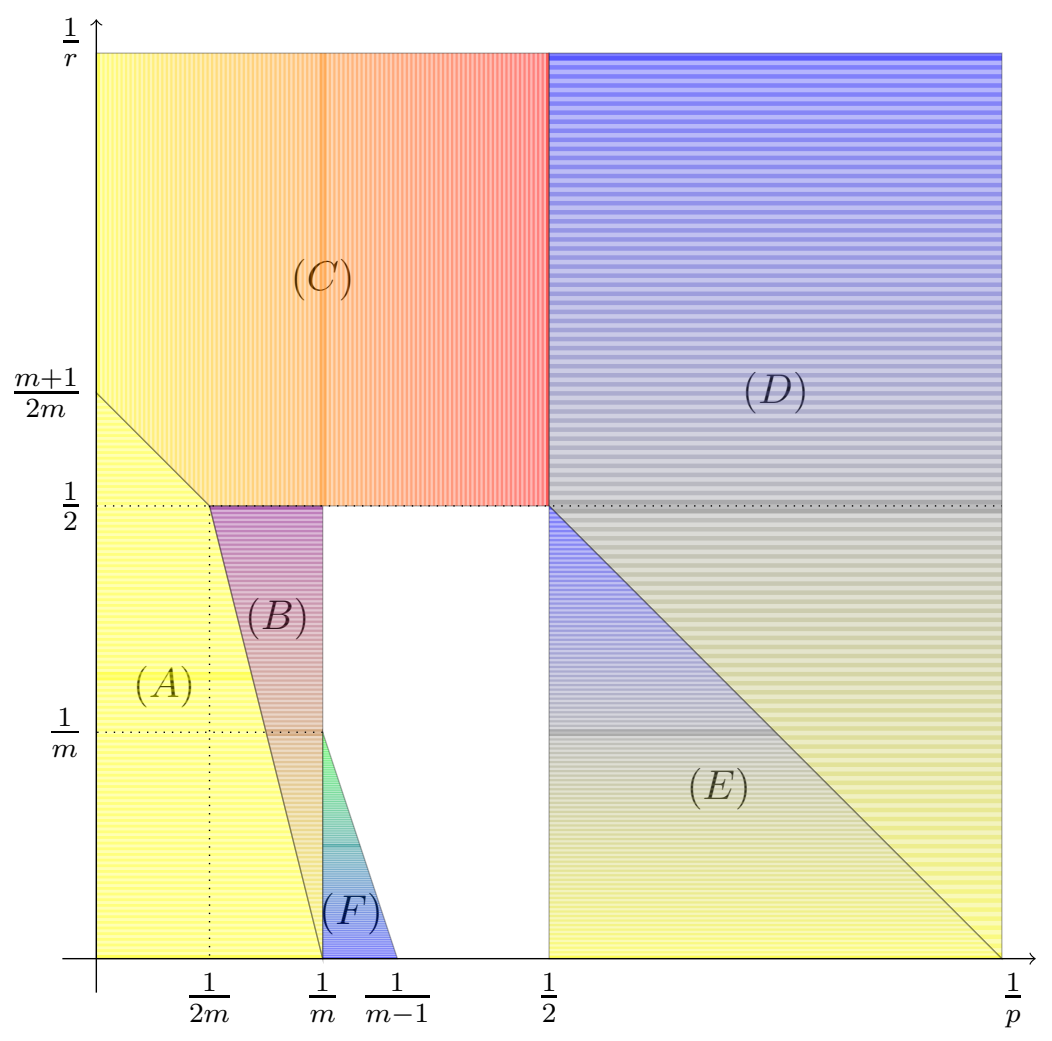

FiguRE 1. Graphical overview of the regions described in Theorem 2.1. consequence of Hölder's inequality,

$$
\begin{aligned}
|P|_{q} \leq\left(\begin{array}{c}
n+m-1 \\
m
\end{array}\right)^{\frac{1}{q}-\frac{1}{r}}|P|_{r} \ll n^{m\left(\frac{1}{q}-\frac{1}{r}\right)}|P|_{r} & \text { if } 1 \leq q \leq r \leq \infty . \\
\|P\|_{\mathcal{P}\left(m \ell_{p}^{n}\right)} \leq n^{m\left(\frac{1}{q}-\frac{1}{p}\right)}\|P\|_{\mathcal{P}\left(m \ell_{q}^{n}\right)} & \text { if } 1 \leq q \leq p \leq \infty .
\end{aligned}
$$

Case $(A)$ is immediate from the Hardy-Littlewood inequalities and cases $(B)$ and $(C)$ also appear in [AP15].

$\bullet(A)$ : Suppose first that $\frac{1}{2} \leq \frac{1}{r} \leq \frac{m+1}{2 m}-\frac{1}{p}$. If $q:=\frac{2 m r}{(m+1) r-2 m}$ then $2 m \leq q \leq p$ and by the Hardy-Littlewood inequality, Theorem $1.2(i i)$, we have

$$
|P|_{r} \ll\|P\|_{\mathcal{P}\left(m \ell_{q}^{n}\right)} \ll\|P\|_{\mathcal{P}\left(m \ell_{p}^{n}\right)} .
$$

Now suppose $\frac{1}{r} \leq \frac{1}{2}$ and $\frac{m}{p} \leq 1-\frac{1}{r}$. If we set $q:=\frac{m r}{r-1}$ then $m \leq q \leq \min \{p, 2 m\}$; then reasoning as before (but using part ( $i$ ) of Theorem 1.2) we can easily reach the same conclusion.

$\bullet(B)$ : Taking $p \leq q=\frac{m r}{r-1}$, by the Hardy-Littlewood inequality, Theorem $1.2(i)$, and (8) it follows

$$
|P|_{r} \ll\|P\|_{\mathcal{P}\left(m \ell_{q}^{n}\right)} \ll\|P\|_{\mathcal{P}\left(m \ell_{p}^{n}\right)} n^{m\left(\frac{1}{p}-\frac{1}{q}\right)}=\|P\|_{\mathcal{P}\left(m \ell_{p}^{n}\right)} n^{\frac{m}{p}+\frac{1}{r}-1} .
$$


For the optimality we can take the polynomial

$$
P=\sum_{j=0}^{k-1} z_{m j+1} \cdots z_{m j+m}, \quad \text { with } \quad k=\left[\frac{n}{m}\right]
$$

it can be seen using Lagrange multipliers and the fact that $p \geq m$, that

$$
\|P\|_{\mathcal{P}\left(m \ell_{p}^{n}\right)}=k\left(\frac{1}{m k}\right)^{\frac{m}{p}} \sim n^{1-\frac{m}{p}}
$$

Then,

$$
n^{\frac{1}{r}} \sim k^{\frac{1}{r}}=|P|_{r} \leq A_{p, r}^{m}(n)\|P\|_{\mathcal{P}\left(m \ell_{p}^{n}\right)} \sim A_{p, r}^{m}(n) n^{1-\frac{m}{p}}
$$

and therefore $n^{\frac{m}{p}+\frac{1}{r}-1} \ll A_{p, r}^{m}$.

$\bullet(C)$ : Suppose $\frac{m+1}{2 m} \leq \frac{1}{r}$ and $\frac{1}{p} \leq \frac{1}{2}$. Using the Bohnenblust-Hille inequality Theorem 1.1, inequalities (7) and (8) we have

$$
\begin{aligned}
|P|_{r} & \ll n^{m\left(\frac{1}{r}-\frac{m+1}{2 m}\right)}|P|_{\frac{2 m}{m+1}} \ll n^{m\left(\frac{1}{r}-\frac{m+1}{2 m}\right)}\|P\|_{\mathcal{P}\left(m \ell_{\infty}^{n}\right)} \\
& \ll n^{m\left(\frac{1}{r}-\frac{m+1}{2 m}\right)} n^{\frac{m}{p}}\|P\|_{\mathcal{P}\left(m \ell_{p}^{n}\right)}=n^{m\left(\frac{1}{p}+\frac{1}{r}-\frac{1}{2}\right)-\frac{1}{2}}\|P\|_{\mathcal{P}\left(m \ell_{p}^{n}\right)} .
\end{aligned}
$$

Suppose $\frac{1}{2} \leq \frac{1}{r} \leq \frac{m+1}{2 m} \leq \frac{1}{p}+\frac{1}{r}$ and let $q:=\frac{2 m r}{(m+1) r-2 m}$. Note that $\max \{2 m, p\} \leq q$. By the Hardy-Littlewood inequality, Theorem 1.2 (ii) and (8) we get

$$
|P|_{r} \ll\|P\|_{\mathcal{P}\left({ }^{m} \ell_{q}^{n}\right)} \ll n^{m\left(\frac{1}{p}-\frac{1}{q}\right)}\|P\|_{\mathcal{P}\left({ }^{m} \ell_{p}^{n}\right)}=n^{m\left(\frac{1}{p}+\frac{1}{r}-\frac{1}{2}\right)-\frac{1}{2}}\|P\|_{\mathcal{P}\left({ }^{m} \ell_{p}^{n}\right)} .
$$

To show that this asymptotic growth is optimal, we consider $P$ an $m$-homogeneous unimodular polynomial as in (41). Then, as $\frac{1}{p} \leq \frac{1}{2}$

$$
n^{\frac{m}{r}} \ll|P|_{r} \leq A_{p, r}^{m}(n)\|P\|_{\mathcal{P}\left(m \ell_{p}^{n}\right)} \ll A_{p, r}^{m}(n) n^{m\left(\frac{1}{2}-\frac{1}{p}\right)+\frac{1}{2}} .
$$

Therefore,

$$
n^{m\left(\frac{1}{p}+\frac{1}{r}-\frac{1}{2}\right)-\frac{1}{2}}=n^{\frac{m}{r}-\left[m\left(\frac{1}{2}-\frac{1}{p}\right)+\frac{1}{2}\right]} \ll A_{p, r}^{m}(n) .
$$

- $(D)$ : We adapt an argument based on [CKP99 used by the authors in the case $p=r=2$ to show the relationship between the Hilbert-Schmidt and supremum norm of a multilinear form on $\ell_{2}^{n}$. If $T$ is the symmetric $m$-linear form associated to $P$, it induces a $(m-1)$-linear mapping $\tilde{T} \in \mathcal{L}\left({ }^{m-1} l_{p}^{n} ;\left(l_{p}^{n}\right)^{*}\right)$, defined by

$$
\tilde{T}\left(x_{1}, \ldots, x_{m-1}\right)(\cdot)=T\left(x_{1}, \ldots, x_{m-1}, \cdot\right) .
$$


Then

$$
\begin{aligned}
|T|_{r}^{r} & =\sum_{i \in \mathcal{M}(m, n)}\left|T\left(e_{i_{1}}, \ldots, e_{i_{m}}\right)\right|^{r} \\
& =\sum_{i \in \mathcal{M}(m-1, n)} \sum_{l=1}^{n}\left|T\left(e_{i_{1}}, \ldots, e_{i_{m-1}}, e_{l}\right)\right|^{r} \\
& \leq \sum_{i \in \mathcal{M}(m-1, n)}\left(\sum_{l=1}^{n}\left|T\left(e_{i_{1}}, \ldots, e_{i_{m-1}}, e_{l}\right)\right|^{p^{\prime}}\right)^{\frac{r}{p}} n^{\frac{r}{p}+1-r} \\
& \leq n^{m-1+\frac{r}{p}+1-r} \sup _{\left\|x_{i}\right\|_{p} \leq 1}\left\|\tilde{T}\left(x_{1}, \ldots, x_{m-1}\right)\right\|_{p^{\prime}}^{r} \\
& =n^{m+\frac{r}{p}-r}\|T\|_{\mathcal{L}\left(m \ell_{p}^{n}\right)}^{r},
\end{aligned}
$$

where in the first inequality we used Hölder inequality in the case $\frac{p^{\prime}}{r} \geq 1$. Then by equations (5) and (6) we have

$$
|P|_{r} \ll n^{\frac{m}{r}+\frac{1}{p}-1}\|P\|_{\ell_{p}^{n}}
$$

For the optimality, we use (44) so, since $1 \leq p \leq 2$ there exists a unimodular polynomial $P$ such that

$$
n^{\frac{m}{r}} \ll|P|_{r} \leq A_{p, r}^{m}(n)\|P\|_{\mathcal{P}\left(\ell_{p}^{n}\right)} \ll A_{p, r}^{m}(n) n^{1-\frac{1}{p}} .
$$

$\bullet(E)$ : Observe that

$$
A_{p, r}^{m}(n)=\left\|i d: \mathcal{P}\left({ }^{m} \ell_{p}^{n}\right) \rightarrow\left(\mathcal{P}\left({ }^{m} \mathbb{C}^{n}\right),|\cdot|_{r}\right)\right\| .
$$

Thus, if $\frac{1}{r}=\frac{\theta}{p^{\prime}}$, for $0<\theta<1$, we can apply complex interpolation to conclude that

$$
A_{p, r}^{m}(n) \leq\left(A_{p, p^{\prime}}^{m}(n)\right)^{\theta}\left(A_{p, \infty}^{m}(n)\right)^{1-\theta} .
$$

Since $1 \leq p \leq 2$, we have by part $(D)$ that $A_{p, p^{\prime}}^{m}(n) \sim n^{\frac{m-1}{p^{\prime}}}$ and also, applying the Cauchy integral formula we deduce that $A_{p, \infty}^{m}(n) \sim 1$. Therefore, we obtain

$$
A_{p, r}^{m}(n) \ll n^{\frac{m-1}{r}} .
$$

For the lower bound, taking a Steiner polynomial $P \in \mathcal{P}\left({ }^{m} \mathbb{C}^{n}\right)$ as in Corollary [1.6 whose associated partial Steiner system has cardinality $\gg n^{m-1}$ and $1 \leq p \leq 2$, then

$$
n^{\frac{m-1}{r}} \ll|P|_{r} \leq A_{p, r}^{m}(n)\|P\|_{\mathcal{P}\left({ }^{m} \ell_{p}^{n}\right)} \ll A_{p, r}^{m}(n) \log \frac{3 p-3}{p}(n) .
$$

Hence, we have that for every $\varepsilon>0$,

$$
n^{\frac{m-1}{r}-\varepsilon} \ll A_{p, r}^{m}(n) .
$$


- $(F)$ : Let $T$ be the symmetric $m$-linear form associated to $P$ and, given $1 \leq i \leq n$, let us define $T_{i} \in \mathcal{L}\left({ }^{m-1} \mathbb{C}^{n}\right)$ as

$$
T_{i}\left(x_{2}, \ldots, x_{m}\right)=T\left(e_{i}, x_{2}, \ldots, x_{m}\right)
$$

Then

$$
\begin{aligned}
|P|_{r}^{r} \sim|T|_{r}^{r} & =\sum_{i \in \mathcal{M}(m, n)}\left|T\left(e_{i_{1}}, \ldots, e_{i_{m}}\right)\right|^{r} \\
& =\sum_{i=1}^{n}\left|T_{i}\right|_{r}^{r} \\
& \ll \sum_{i=1}^{n}\left\|T_{i}\right\|_{\mathcal{L}\left(m-1 \ell_{p}^{n}\right)}^{r} \\
& \leq n\|T\|_{\mathcal{L}\left(m \ell_{p}^{n}\right)}^{r} \sim n\|P\|_{\mathcal{P}\left(m \ell_{p}^{n}\right)}^{r},
\end{aligned}
$$

where we have used in (9) the fact that $A_{p, r}^{m-1}(n) \sim 1$ for this range of $p$ and $r$. Therefore

$$
|P|_{r} \ll n^{\frac{1}{r}}\|P\|_{\mathcal{P}\left(m \ell_{p}^{n}\right)} .
$$

For the lower bound, let $P=\sum_{j=1}^{k} z_{m j+1} \cdots z_{m j+m}$ as in part $(B)$, then since $p \geq m$ (in region $(F)$ ), we have that $\|P\|_{\mathcal{P}\left(m \ell_{p}^{n}\right)} \sim 1$ and thus

$$
n^{\frac{1}{r}} \sim|P|_{r} \ll A_{p, r}^{m}(n)\|P\|_{\mathcal{P}\left(m \ell_{p}^{n}\right)} \sim A_{p, r}^{m}(n) .
$$

For $2 \leq p \leq m, 2 \leq r<\infty$ and $\left(\frac{1}{p}, \frac{1}{r}\right) \notin(F)$ we could have used interpolation (in vertical direction, as we did in the proof of part $(E)$ of Theorem 2.1) to obtain effective upper bounds for $A_{p, r}^{m}$. We choose not to state them explicitly since we believe these estimates are suboptimal.

2.1. An application to interpolation of tensor products of Banach spaces. Kouba in [Kou91] (see also [DM03, DM00]) proved a remarkable result on complex interpolation of injective tensor products of Banach spaces, which implies that for $L_{p}$-spaces,

$$
\left[L_{p_{0}} \otimes_{\varepsilon} L_{q_{0}}, L_{p_{1}} \otimes_{\varepsilon} L_{q_{1}}\right]_{\theta}=L_{p} \otimes_{\varepsilon} L_{q}
$$

for $0<\theta<1,1 \leq p_{0}, q_{0}, p_{1}, q_{1} \leq 2, \frac{1}{p}=\frac{1-\theta}{p_{0}}+\frac{\theta}{p_{1}}$ and $\frac{1}{q}=\frac{1-\theta}{q_{0}}+\frac{\theta}{q_{1}}$.

We will show that Theorem 2.1 implies that a similar statement does not hold for the $m$-fold injective tensor, $m>2$. Indeed, we will show that the following problem has a negative answer. 
Problem 2.2. Let $1 \leq p_{0}, p_{1} \leq 2,0<\theta<1$ and $n, m \in \mathbb{N}$ and consider

$$
\iota_{\theta}: \otimes_{i=1, \varepsilon}^{m}\left[\ell_{p_{0}}^{n}, \ell_{p_{1}}^{n}\right]_{\theta} \longrightarrow\left[\otimes_{i=1, \varepsilon}^{m} \ell_{p_{0}}^{n}, \otimes_{i=1, \varepsilon}^{m} \ell_{p_{1}}^{n}\right]_{\theta}
$$

the natural inclusion. Is there any constant $C>0$ independent of $n$ such that

$$
\left\|\iota_{\theta}\right\| \leq C ?
$$

We are sincerely grateful to Jorge Tomás Rodriguez, who gently gave us the idea of the following family of counterexamples.

Remark 2.3. The answer to the question in Problem 2.2 is negative in general. In particular, for $m \geq 3, r>m,(m-1) r^{\prime} \leq p_{1}^{\prime}<m, m r^{\prime} \leq p_{0}^{\prime}$ and $\theta$ such that $\frac{1}{p^{\prime}}=\frac{1-\theta}{p_{0}^{\prime}}+\frac{\theta}{p_{1}^{\prime}} \leq m$ there is no $C>0$ independent of $n$ such that $\left\|\iota_{\theta}\right\| \leq C$.

Proof. Observe that $\left(\frac{1}{p_{0}^{\prime}}, \frac{1}{r}\right) \in(A)$ and $\left(\frac{1}{p_{1}^{\prime}}, \frac{1}{r}\right) \in(F)$ with $p_{1}^{\prime}>m$. Assuming a positive answer to Problem 2.2, we would have that for, $\frac{1}{p^{\prime}}=\frac{1-\theta}{p_{0}^{\prime}}+\frac{\theta}{p_{1}^{\prime}}, \theta \in(0,1)$,

$$
A_{p, r}^{m}(n) \leq n^{\frac{1-\theta}{r}}
$$

If $\theta$ fulfills the condition $\frac{1}{p^{\prime}}=\frac{1-\theta}{p_{0}}+\frac{\theta}{p_{1}} \leq m$ we have $\left(p^{\prime}, r\right) \in(F)$, this contradicts the lower bound from region $(F)$ in Theorem 2.1 .

In [BPR18] the authors gave a similar result independently and with a different approach. Observe that the question in Problem 2.2 still unanswered when both $2 \leq p_{0}^{\prime}, p_{1}^{\prime} \leq m$ and $p_{0}^{\prime} \neq p_{1}^{\prime}$. In the case the answer is affirmative for these parameters, this would allow us to prove the remaining cases in Theorem 2.1 .

2.2. Asymptotic estimates for $B_{r, p}^{m}(n)$. We now present the correct asymptotic behavior for the constants $B_{r, p}^{m}(n)$ defined in Problem 1.3. These estimates will be useful in the next section for the applications.

Proposition 2.4. Let $B_{r, p}^{m}(n)$ be the smallest constant such that for every $m$-homogeneous polynomial $P$ in $n$ complex variables, $\|P\|_{\left.\mathcal{P}^{(m} \ell_{p}^{n}\right)} \leq B_{r, p}^{m}(n)|P|_{r}$. We have

$$
B_{r, p}^{m}(n) \sim \begin{cases}1 & \text { for } r \leq p^{\prime} \\ n^{m\left(1-\frac{1}{p}-\frac{1}{r}\right)} & \text { for } r \geq p^{\prime}\end{cases}
$$


Proof. Let $n, m \in \mathbb{N}$ and $1 \leq p, r \leq \infty$. Let $P=\sum_{\alpha \in \Lambda(m, n)} a_{\alpha} z^{\alpha}$ be an $m$-homogeneous polynomial in $n$ variables. Suppose first that $r \leq p^{\prime}$. Then

$$
\begin{aligned}
\|P\|_{\mathcal{P}\left(m \ell_{p}^{n}\right)} & =\sup _{z \in B_{\ell_{p}^{n}}}\left|\sum_{\alpha \in \Lambda(m, n)} a_{\alpha} z^{\alpha}\right| \\
& \leq \sup _{z \in B_{\ell_{p}^{n}}}\left(\sum_{\alpha \in \Lambda(m, n)}\left|a_{\alpha}\right|^{p^{\prime}}\right)^{\frac{1}{p^{\prime}}}\left(\sum_{\alpha \in \Lambda(m, n)}\left|z^{\alpha}\right|^{p}\right)^{\frac{1}{p}} \\
& \leq|P|_{p^{\prime}} \sup _{z \in B_{\ell_{p}^{n}}}\left(\sum_{\mathbf{i} \in \mathcal{M}(m, n)}\left|z_{\mathbf{i}}\right|^{p}\right)^{\frac{1}{p}} \\
& =|P|_{p^{\prime}} \sup _{z \in B_{\ell_{p}^{n}}}\left(\sum_{k=1}^{n}\left|z_{k}\right|^{p}\right)^{\frac{m}{p}} \\
& =|P|_{p^{\prime}} \\
& \leq|P|_{r} .
\end{aligned}
$$

On the other hand, if $r \geq p^{\prime}$,

$$
\begin{aligned}
\|P\|_{\mathcal{P}\left(m \ell_{p}^{n}\right)} & \leq|P|_{p^{\prime}} \\
& \leq|P|_{r} n^{m\left(\frac{1}{p^{\prime}}-\frac{1}{r}\right)} \\
& =|P|_{r} n^{m\left(1-\frac{1}{p}-\frac{1}{r}\right)} .
\end{aligned}
$$

To study lower bounds, let us take the polynomial $P(z)=\sum_{\mathbf{j} \in \mathcal{J}(m, n)} z_{\mathbf{j}}$. Note that $|P|_{r} \sim n^{\frac{m}{r}}$ and

$$
\begin{aligned}
\|P\|_{\mathcal{P}\left(m \ell_{p}^{n}\right)} & =\sup _{z \in B_{\ell_{p}^{n}}}\left|\sum_{\mathbf{j} \in \mathcal{J}(m, n)} z_{\mathbf{j}}\right| \\
& \geq\left|\sum_{\mathbf{j} \in \mathcal{J}(m, n)} n^{-\frac{m}{p}}\right| \quad \text { taking } z=\overbrace{\left(\frac{1}{n^{\frac{1}{p}}}, \ldots, \frac{1}{n^{\frac{1}{p}}}\right)}^{m} \\
& \sim n^{m\left(1-\frac{1}{p}\right)} .
\end{aligned}
$$

Therefore $B_{r, p}^{m}(n) \gg n^{m\left(1-\frac{1}{r}-\frac{1}{p}\right)}$. 


\section{Mixed UnCONDITIONAL BASIS CONSTANT FOR HOMOGENEOUS POLYNOMIALS ON $\ell_{p}$ SPACES}

Here we will study the asymptotic growth of $\chi_{p, q}\left(\mathcal{P}\left({ }^{m} \mathbb{C}^{n}\right)\right)$ for fixed $1 \leq q, p \leq \infty$ and $m \in \mathbb{N}$ as $n$ tends to infinity (see Definition 1.4).

The following result shows that, in order to study the asymptotic behavior of the mixed unconditional constants of $\mathcal{P}\left({ }^{m} \mathbb{C}^{n}\right)$, it is enough to understand what happens with the monomial basis $\left(z_{\mathbf{j}}\right)_{\mathbf{j} \in \mathcal{J}(m, n)}$. These can be seen as a sort extension of a result of Pisier and Schütt [Pis78, Sch78] (see also [DDGM01, DF11, CG11]).

Theorem 3.1. We have the following relation:

$$
\chi_{p, q}\left(\mathcal{P}\left({ }^{m} \mathbb{C}^{n}\right)\right) \leq \chi_{p, q}\left(\left(z_{\mathbf{j}}\right)_{\mathbf{j} \in \mathcal{J}(m, n)}\right) \leq 2^{m} \chi_{p, q}\left(\mathcal{P}\left({ }^{m} \mathbb{C}^{n}\right)\right)
$$

Our proof relies on Szarek's approach [Sza81] combined with the following inequality due to Bayart Bay02 (see also [Wei80, DM15]).

Lemma 3.2 (Bayart's inequality). Let $P(z)=\sum_{\mathbf{j} \in \mathcal{J}(m, n)} c_{\mathbf{j}} z_{\mathbf{j}}$ be an m-homogeneous polynomial in n-variables. Then

$$
\left(\sum_{\mathbf{j} \in \mathcal{J}(m, n)}\left|c_{\mathbf{j}}\right|^{2}\right)^{1 / 2} \leq 2^{m / 2} \int_{\mathbb{T}^{n}}|P(w)| d w
$$

where $\mathbb{T}^{n}$ stands for the $n$-dimensional torus and $d w$ is the normalized Lebesgue measure on $\mathbb{T}^{n}$.

Before giving the proof we define the following operator. For any $w=\left(w_{1} \ldots, w_{n}\right) \in \mathbb{T}^{n}$ and any $1 \leq p \leq \infty$ we define the operator

$$
\begin{aligned}
T_{w}^{p}: \mathcal{P}\left({ }^{m} \ell_{p}^{n}\right) & \longrightarrow \mathcal{P}\left({ }^{m} \ell_{p}^{n}\right) \\
\sum_{\mathbf{j} \in \mathcal{J}(m, n)} a_{\mathbf{j}} z_{\mathbf{j}} & \longmapsto \sum_{\mathbf{j} \in \mathcal{J}(m, n)} a_{\mathbf{j}} z_{\mathbf{j}} w_{\mathbf{j}},
\end{aligned}
$$

which clearly has norm one.

We also need a remark whose proof is straightforward from Definition 1.4 .

Remark 3.3. Let $\left(P_{i}\right)_{i \in \Lambda}$ be a basis for $\mathcal{P}\left({ }^{m} \mathbb{C}^{n}\right)$ and $\left(P_{i}^{\prime}\right)_{i \in \Lambda}$ its dual basis (i.e., $\left.\left\langle P_{i}^{\prime}, P_{k}\right\rangle=\delta_{i . k}\right)$. For $1 \leq q, p \leq \infty$ and $n, m \in \mathbb{N}, \chi_{p, q}\left(\left(P_{i}\right)_{i \in \Lambda}\right)$ is exactly the best constant $C>0$ such that

$$
\sum_{i \in \Lambda}\left|\left\langle P_{i}^{\prime}, Q\right\rangle\left\langle Q^{\prime}, P_{i}\right\rangle\right| \leq C\|Q\|_{\mathcal{P}\left(m \ell_{p}^{n}\right)}\left\|Q^{\prime}\right\|_{\mathcal{P}\left(m \ell_{q}^{n}\right)^{\prime}}
$$

for every $Q \in \mathcal{P}\left({ }^{m} \mathbb{C}^{n}\right)$ and $Q^{\prime} \in \mathcal{P}\left({ }^{m} \mathbb{C}^{n}\right)^{\prime}$. 
Proof of Theorem 3.1. Let $\left(P_{i}\right)_{i \in \Lambda}$ be a basis for $\mathcal{P}\left({ }^{m} \mathbb{C}^{n}\right)$ and $\left(P_{i}^{\prime}\right)_{i \in \Lambda}$ its dual basis. Consider $Q \in \mathcal{P}\left({ }^{m} \mathbb{C}^{n}\right)$ and $Q^{\prime} \in \mathcal{P}\left({ }^{m} \mathbb{C}^{n}\right)^{\prime}$. Since $1=\left|\left\langle z_{\mathbf{j}}^{\prime}, z_{\mathbf{j}}\right\rangle\right|=\left|\sum_{i \in \Lambda}\left\langle z_{\mathbf{j}}^{\prime}, P_{i}\right\rangle\left\langle P_{i}^{\prime}, z_{\mathbf{j}}\right\rangle\right|$, we have

$$
\begin{aligned}
\sum_{\mathbf{j} \in \mathcal{J}(m, n)} \mid\left\langle Q^{\prime}, z_{\mathbf{j}}\right\rangle & \left\langle z_{\mathbf{j}}^{\prime}, Q\right\rangle\left|=\sum_{\mathbf{j} \in \mathcal{J}(m, n)}\right|\left\langle Q^{\prime}, z_{\mathbf{j}}\right\rangle\left\langle z_{\mathbf{j}}^{\prime}, Q\right\rangle|| \sum_{i \in \Lambda}\left\langle z_{\mathbf{j}}^{\prime}, P_{i}\right\rangle\left\langle P_{i}^{\prime}, z_{\mathbf{j}}\right\rangle \mid \\
& \leq \sum_{i \in \Lambda} \sum_{\mathbf{j} \in \mathcal{J}(m, n)}\left|\left\langle Q^{\prime}, z_{\mathbf{j}}\right\rangle\left\langle z_{\mathbf{j}}^{\prime}, Q\right\rangle\left\langle z_{\mathbf{j}}^{\prime}, P_{i}\right\rangle\left\langle P_{i}^{\prime}, z_{\mathbf{j}}\right\rangle\right| \\
& \leq \sum_{i \in \Lambda}\left(\sum_{\mathbf{j} \in \mathcal{J}(m, n)}\left|\left\langle Q^{\prime}, z_{\mathbf{j}}\right\rangle\left\langle z_{\mathbf{j}}^{\prime}, P_{i}\right\rangle\right|^{2}\right)^{\frac{1}{2}}\left(\sum_{\mathbf{j} \in \mathcal{J}(m, n)}\left|\left\langle z_{\mathbf{j}}^{\prime}, Q\right\rangle\left\langle P_{i}^{\prime}, z_{\mathbf{j}}\right\rangle\right|^{2}\right)^{\frac{1}{2}} \\
& \leq \sum_{i \in \Lambda} 2^{m / 2} \int_{\mathbb{T}^{n}}\left|\sum_{\mathbf{j} \in \mathcal{J}(m, n)}\left\langle Q^{\prime}, z_{\mathbf{j}}\right\rangle\left\langle z_{\mathbf{j}}^{\prime}, P_{i}\right\rangle w_{\mathbf{j}}\right| d w \cdot 2^{m / 2} \int_{\mathbb{T}^{n}}\left|\sum_{\mathbf{j} \in \mathcal{J}(m, n)}\left\langle z_{\mathbf{j}}^{\prime}, Q\right\rangle\left\langle P_{i}^{\prime}, z_{\mathbf{j}}\right\rangle \tilde{w}_{\mathbf{j}}\right| d \tilde{w} \\
& =\sum_{i \in \Lambda} 2^{m} \int_{\mathbb{T}^{n}}\left|\left\langle\left(T_{w}^{q}\right)^{*}\left(Q^{\prime}\right), P_{i}\right\rangle\right| d w \cdot \int_{\mathbb{T}^{n}}\left|\left\langle P_{i}^{\prime}, T_{\tilde{w}}^{p}(Q)\right\rangle\right| d \tilde{w} \\
& =2^{m} \int_{\mathbb{T}^{n} \times \mathbb{T}^{n}} \sum_{i \in \Lambda}\left|\left\langle\left(T_{w}^{q}\right)^{*}\left(Q^{\prime}\right), P_{i}\right\rangle\left\langle P_{i}^{\prime}, T_{\tilde{w}}^{p}(Q)\right\rangle\right| d w d \tilde{w} \\
& \leq 2^{m} \int_{\mathbb{T}^{n} \times \mathbb{T}^{n}} \chi_{p, q}\left(\left(P_{i}\right)_{i \in \Lambda}\right)\left\|\left(T_{w}^{q}\right)^{*}\left(Q^{\prime}\right)\right\|_{\mathcal{P}\left(m \ell_{q}^{n}\right)^{\prime}}\left\|T_{\tilde{w}}^{p}(Q)\right\|_{\mathcal{P}\left(m \ell_{p}^{n}\right)} d w d \tilde{w} \\
& \leq 2^{m} \chi_{p, q}\left(\left(P_{i}\right)_{i \in \Lambda}\right)\left\|Q^{\prime}\right\|_{\mathcal{P}\left(m \ell_{q}^{n}\right)^{\prime}}\|Q\|_{\mathcal{P}\left(m \ell_{p}^{n}\right),}
\end{aligned}
$$

where we applied Cauchy-Schwarz for the second inequality, Bayart's inequality (10) for the third one and Remark 3.3 for the basis $\left(P_{i}\right)$ for the next to last inequality. Using Remark 3.3 again but for the monomial basis $\left(z_{\mathbf{j}}\right)_{\mathbf{j} \in \mathcal{J}(m, n)}$ we have that

$$
\chi_{p, q}\left(\left(z_{\mathbf{j}}\right)_{\mathbf{j} \in \mathcal{J}(m, n)}\right) \leq 2^{m} \chi_{p, q}\left(\left(P_{i}\right)_{i \in \Lambda}\right) .
$$

Since $\left(P_{i}\right)_{i \in \Lambda}$ is an arbitrary basis of $\mathcal{P}\left({ }^{m} \mathbb{C}^{n}\right)$ we have

$$
\chi_{p, q}\left(\mathcal{P}\left({ }^{m} \mathbb{C}^{n}\right)\right) \leq \chi_{p, q}\left(\left(z_{\mathbf{j}}\right)_{\mathbf{j} \in \mathcal{J}(m, n)}\right) \leq 2^{m} \chi_{p, q}\left(\mathcal{P}\left({ }^{m} \mathbb{C}^{n}\right)\right),
$$

which concludes the proof.

We now present some estimates for the asymptotic behavior of the mixed- $(p, q)$ unconditional constant of $\mathcal{P}\left({ }^{m} \mathbb{C}^{n}\right)$. Note that in the case $q=p$ we recover the results from [DDGM01]. 


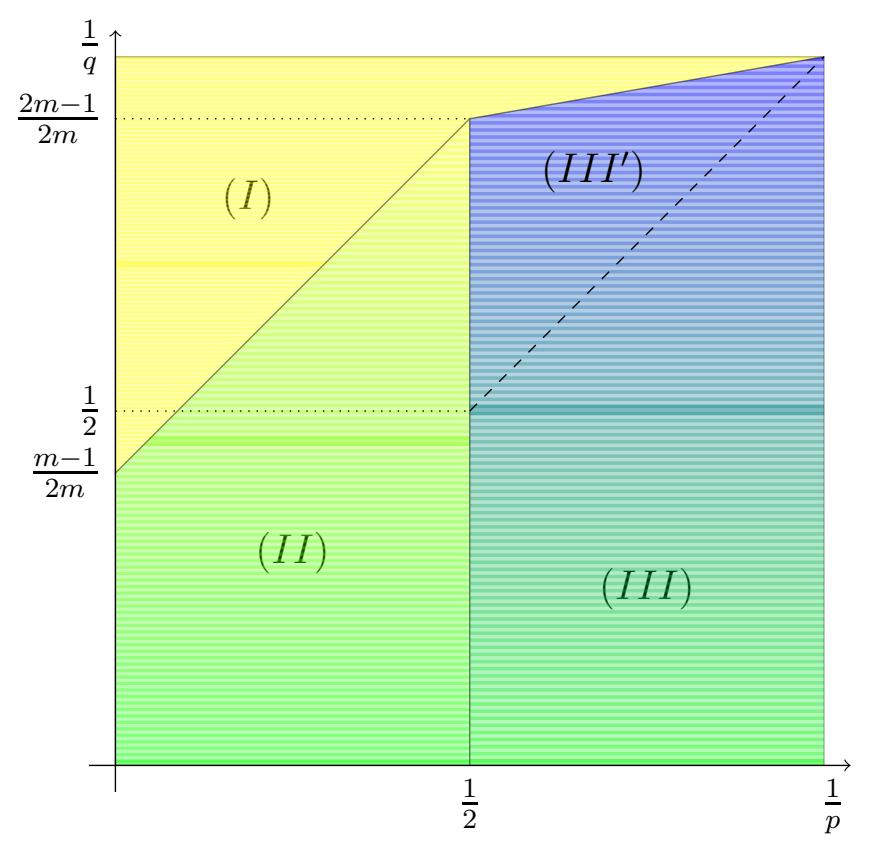

Figure 2. Graphical overview of the mixed unconditional constant described in Theorem 3.4 .

\section{Theorem 3.4.}

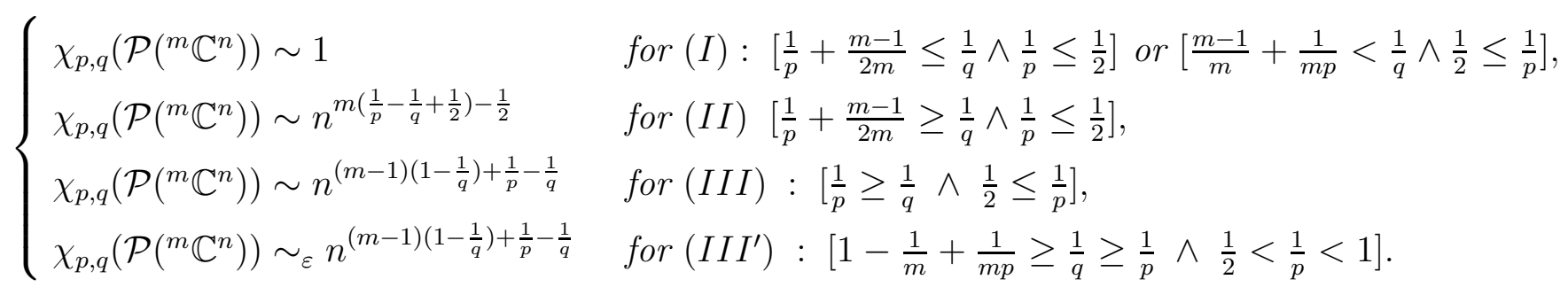

where $\chi_{p, q}\left(\mathcal{P}\left({ }^{m} \mathbb{C}^{n}\right)\right) \sim_{\varepsilon} n^{(m-1)\left(1-\frac{1}{q}\right)+\frac{1}{p}-\frac{1}{q}}$ means that

$$
n^{(m-1)\left(1-\frac{1}{q}\right)+\frac{1}{p}-\frac{1}{q}} \ll \chi_{p, q}\left(\mathcal{P}\left(^{m} \mathbb{C}^{n}\right)\right) \ll n^{(m-1)\left(1-\frac{1}{q}\right)+\frac{1}{p}-\frac{1}{q}+\varepsilon},
$$

for every $\varepsilon>0$.

Moreover, in $\left(I I I^{\prime}\right)$ for every $\lambda>\frac{1}{p}$ we have

$$
n^{(m-1)\left(1-\frac{1}{q}\right)+\frac{1}{p}-\frac{1}{q}} \ll \chi_{p, q}\left(\mathcal{P}\left(^{m} \mathbb{C}^{n}\right)\right) \ll \log (n)^{m\left(\frac{1}{q}-\frac{1}{p}\right)+\left(\lambda+\frac{1}{p}\right) \frac{m^{2}}{m-1} \frac{p-q}{q(p-1)}} n^{(m-1)\left(1-\frac{1}{q}\right)+\frac{1}{p}-\frac{1}{q}} .
$$

To prove the theorem we need a lemma and also to recall some results on monomial convergence.

Lemma 3.5. Let $1 \leq q, p \leq \infty$, then we have

$$
\chi_{p, q}\left(\mathcal{P}\left({ }^{m} \mathbb{C}^{n}\right)\right) \ll B_{r, q}^{m}(n) A_{p, r}^{m}(n) \quad \text { for every } 1 \leq r \leq \infty
$$


Proof. Let $P(z)=\sum_{\mathbf{j} \in \mathcal{J}(m, n)} c_{\mathbf{j}} z_{\mathbf{j}}$ be an $m$-homogeneous polynomial in $n$ variables and $\left(\theta_{\mathbf{j}}\right) \mathbf{j} \in \mathcal{J}(m, n)$ be a sequence of complex numbers of modulus one, then

$$
\left\|\sum_{\mathbf{j} \in \mathcal{J}(m, n)} \theta_{\mathbf{j}} c_{\mathbf{j}} z_{\mathbf{j}}\right\|_{\mathcal{P}\left(m \ell_{q}^{n}\right)} \leq B_{r, q}^{m}(n)\left(\sum_{\mathbf{j} \in \mathcal{J}(m, n)}\left|c_{\mathbf{j}}\right|^{r}\right)^{\frac{1}{r}} \leq B_{r, q}^{m}(n) A_{q, r}^{m}(n)\left\|\sum_{\mathbf{j} \in \mathcal{J}(m, n)} c_{\mathbf{j}} z_{\mathbf{j}}\right\|_{\mathcal{P}\left(m \ell_{p}^{n}\right)},
$$

for every $1 \leq r \leq \infty$. Thus $\chi_{p, q}\left(\left(z_{\mathbf{j}}\right)_{\mathbf{j} \in \mathcal{J}(m, n)}\right) \leq B_{r, q}^{m}(n) A_{p, r}^{m}(n)$ and the result follows from Theorem 3.1 .

The set of monomial convergence for $m$-homogeneous polynomials over the domain $\ell_{p}$, denoted by $\operatorname{mon}\left(\mathcal{P}\left({ }^{m} \ell_{p}\right)\right)$, is defined as

$$
\operatorname{mon}\left(\left(\mathcal{P}\left({ }^{m} \ell_{p}\right)\right):=\left\{z \in \ell_{p}: \sum_{\alpha \in \mathbb{N}_{0}^{\mathbb{N}}}\left|c_{\alpha}(P) z^{\alpha}\right|<\infty, \text { for all } P \in \mathcal{P}\left({ }^{m} \ell_{p}\right)\right)\right\}
$$

See [DMP09, BDS15] and the references therein for what is known about these sets. There is a strong relation between monomial convergence and mixed unconditionality. Let $X, Y$ be Banach sequence spaces. We denote by $X_{n}$ and $Y_{n}$ the $n$-dimensional subspaces given by the span of the first $n$ canonical vectors in $X$ and $Y$ respectively. The following result is an immediate consequence of [DMP09, Theorem 5.2].

Theorem 3.6. Let $X, Y$ be Banach sequence spaces. Then the following are equivalent

i) $Y \subset \operatorname{mon}\left(\mathcal{P}\left({ }^{m} X\right)\right)$,

ii) there exists $C=C(m)>0$ such that for every $m$-homogeneous polynomial in $n$ variables $P(z)=\sum_{\mathbf{j} \in \mathcal{J}(m, n)} c_{\mathbf{j}} z_{\mathbf{j}}$, and every $u \in B_{Y_{n}}$

$$
\sum_{\mathbf{j} \in \mathcal{J}(m, n)}\left|c_{\mathbf{j}} u_{\mathbf{j}}\right| \leq C\|P\|_{\mathcal{P}\left({ }^{m} X_{n}\right)}
$$

In particular,

$$
\ell_{q} \subset \operatorname{mon}\left(\mathcal{P}\left({ }^{m} \ell_{p}\right)\right) \text { if and only if } \chi_{p, q}\left(\mathcal{P}\left({ }^{m} \mathbb{C}^{n}\right)\right) \sim 1
$$

Let $\mathbf{p}=\left(p_{j}\right)_{j \in \mathbb{N}}$ be the sequence of prime numbers. For a real function $f$, we define $f(\mathbf{p}):=$ $\left(f\left(p_{j}\right)\right)_{j \in \mathbb{N}}$, and we denote by $f(\mathbf{p}) \cdot \ell_{p}$ the set $\left\{\left(f\left(p_{j}\right) x_{j}\right)_{j \in \mathbb{N}}: x \in \ell_{p}\right\}$.

Lemma 3.7. Let $\frac{1}{2} \leq \frac{1}{p}$ and $\frac{1}{q_{m}}=1-\frac{1}{m}+\frac{1}{m p}$. For $\lambda>\frac{1}{p}$, we define the sequence $w_{\lambda}:=\left(w_{\lambda}(j)\right)_{j \in \mathbb{N}}$ where $w_{\lambda}(j)=\log \left(p_{j}\right)^{q_{m} \lambda+1}$ and define the Lorentz Banach sequence space

$$
d\left(w_{\lambda}, q_{m}\right)=\left\{y=\left(y_{j}\right)_{j \in \mathbb{N}}:\|y\|_{d\left(w_{\lambda}, q_{m}\right)}:=\left(\sum_{j}\left|y_{j}^{*}\right|^{q_{m}} w_{\lambda}(j)\right)^{1 / q_{m}}<\infty\right\},
$$


where $y^{*}$ denotes the decreasing rearrangement of $y$. Then,

$$
d\left(w_{\lambda}, q_{m}\right) \subset \operatorname{mon}\left(\mathcal{P}\left({ }^{m} \ell_{p}\right)\right)
$$

Proof. Note that $y \in \operatorname{mon}\left(\mathcal{P}\left({ }^{m} \ell_{p}\right)\right)$ if and only if its decreasing rearrangement $y^{*}$ belongs to $\operatorname{mon}\left(\mathcal{P}\left({ }^{m} \ell_{p}\right)\right)$. In [BDS15, Theorem 5.3] it was proved that for any $\varepsilon>\frac{1}{p}$ we have,

$$
\frac{1}{\mathbf{p}^{\sigma_{m}} \log (\mathbf{p})^{\varepsilon}} \cdot \ell_{p} \subset \operatorname{mon}\left(\mathcal{P}\left({ }^{m} \ell_{p}\right)\right)
$$

where $\sigma_{m}=\left(\frac{m-1}{m}\right)\left(1-\frac{1}{p}\right)$. We will show that for every $y \in d\left(w_{\lambda}, q_{m}\right)$ we have that $y^{*} \in$ $\frac{1}{\mathbf{p}^{\sigma m} \log (\mathbf{p})^{\varepsilon_{0}}} \cdot \ell_{p}$ for some $\varepsilon_{0}>\frac{1}{p}$.

Notice that

$$
\log \left(p_{n}\right)^{\lambda q_{m}+1}\left(y_{n}^{*}\right)^{q_{m}} \ll \frac{1}{n}
$$

indeed, if not there is a subsequence $\left(n_{k}\right)$ and constants $C_{k} \rightarrow \infty$ such that

$$
\log \left(p_{n_{k}}\right)^{\lambda q_{m}+1}\left(y_{n_{k}}^{*}\right)^{q_{m}} \geq C_{k} \frac{1}{n_{k}}
$$

By the Prime Number Theorem $\left(p_{n} \sim n \log (n)\right)$ and some elementary computations, we have that for sufficiently large $k, \log \left(p_{j}\right) / \log \left(p_{n_{k}}\right) \geq 1 / 2$ if $j \geq n_{k} / 2$. Thus,

$$
\begin{aligned}
\sum_{j=1}^{n_{k}} \log \left(p_{j}\right)^{\lambda q_{m}+1}\left(y_{j}^{*}\right)^{q_{m}} & \geq\left(y_{n_{k}}^{*}\right)^{q_{m}} \sum_{n_{k} / 2 \leq j \leq n_{k}} \log \left(p_{j}\right)^{\lambda q_{m}+1} \\
& \geq \frac{1}{2}\left(y_{n_{k}}^{*}\right)^{q_{m}} \log \left(p_{n_{k}}\right)^{\lambda q_{m}+1} \cdot \frac{n_{k}}{2} \\
& \geq \frac{C_{k}}{4} \rightarrow \infty,
\end{aligned}
$$

which is a contradiction since $y \in d\left(w_{\lambda}, q_{m}\right)$. Therefore, by (14),

$$
y_{n}^{*} \ll \frac{1}{n^{\frac{1}{q_{m}}}} \frac{1}{\log \left(p_{n}\right)^{\frac{1}{q_{m}}+\lambda}} \leq \frac{1}{n^{\frac{1}{q_{m}}}} \frac{1}{\log (n)^{\frac{1}{q_{m}}+\lambda}} .
$$

If we take $\varepsilon_{0}, \tilde{\lambda}$ such that $\frac{1}{p}<\varepsilon_{0}<\tilde{\lambda}<\lambda$, and use the Prime Number Theorem we have

$$
\begin{aligned}
p_{n}^{\sigma_{m}} \log \left(p_{n}\right)^{\varepsilon_{0}} y_{n}^{*} & \ll \frac{p_{n}^{\sigma_{m}} \log \left(p_{n}\right)^{\varepsilon_{0}}}{n^{\frac{1}{q_{m}}} \log (n)^{\frac{1}{q_{m}}+\lambda}} \\
& \ll \frac{n^{\sigma_{m}} \log (n)^{\sigma_{m}+\tilde{\lambda}}}{n^{\frac{1}{q_{m}}} \log (n)^{\frac{1}{q_{m}}+\lambda}} \\
& =n^{-\frac{1}{p}} \log (n)^{-\frac{1}{p}+\tilde{\lambda}-\lambda} .
\end{aligned}
$$

Since the sequence $\left(n^{-\frac{1}{p}} \log (n)^{-\frac{1}{p}+\tilde{\lambda}-\lambda}\right)_{n \geq 1}$ is in $\ell_{p}$, we conclude the proof. 
Lemma 3.8. Let $\frac{1}{2} \leq \frac{1}{p}$ and $\frac{1}{q_{m}}=1-\frac{1}{m}+\frac{1}{m p}$. Then if $\lambda>\frac{1}{p}$,

$$
\chi_{p, q_{m}}\left(\mathcal{P}\left({ }^{m} \mathbb{C}^{n}\right)\right) \ll \log (n)^{m\left(\lambda+\frac{1}{q_{m}}\right)} .
$$

Proof. Let $\lambda>\tilde{\lambda}>\frac{1}{p}$. If $u \in B_{\ell_{q m}^{n}}$ then, by the Prime Number Theorem, we know that

$$
\|u\|_{d\left(w_{\tilde{\lambda}}, q_{m}\right)_{n}} \leq \log \left(p_{n}\right)^{\tilde{\lambda}+\frac{1}{q_{m}}} \ll \log (n)^{\lambda+\frac{1}{q_{m}}} .
$$

For every $m$-homogeneous polynomial in $n$ variables $P(z)=\sum_{\mathbf{j} \in \mathcal{J}(m, n)} c_{\mathbf{j}} z_{\mathbf{j}}$ and every $u \in B_{\ell_{q_{m}}^{n}}$, by Lemma 3.7 and Theorem 3.6, we have that

$$
\begin{aligned}
\sum_{\mathbf{j} \in \mathcal{J}(m, n)}\left|c_{\mathbf{j}} u_{\mathbf{j}}\right| & \leq C\|u\|_{d\left(w_{\tilde{\lambda}}, q_{m}\right)_{n}}^{m}\|P\|_{\mathcal{P}\left(m \ell_{p}^{n}\right)} \\
& \ll \log (n)^{m\left(\lambda+\frac{1}{q_{m}}\right)}\|P\|_{\mathcal{P}\left(\ell_{p}^{n}\right)},
\end{aligned}
$$

and from this inequality it is easy to conclude the proof.

We now prove Theorem 3.4.

Proof of Theorem 3.4. The proof is divided in cases.

-(I) : Let $p \geq 2$. By [DMP09, Example 4.6] we know that $\ell_{q_{m}} \subset \operatorname{mon}\left(\mathcal{P}\left({ }^{m} \ell_{p}\right)\right)$ where

$$
\frac{1}{q_{m}}=\frac{1}{p}+\frac{m-1}{2 m} .
$$

On the hand, if $p \leq 2$, by BDS15, Theorem 5.1] we know that $\ell_{q_{m}-\varepsilon} \subset \operatorname{mon}\left(\mathcal{P}\left({ }^{m} \ell_{p}\right)\right)$ for every $\varepsilon>0$ where

$$
\frac{1}{q_{m}}=\frac{m-1}{m}+\frac{1}{m p}
$$

Therefore, by the statement in (13) and monotonicity we known that $\chi_{p, q}\left(\mathcal{P}\left({ }^{m} \mathbb{C}^{n}\right)\right) \sim 1$ in region

$$
(I):\left[\frac{1}{p}+\frac{m-1}{2 m} \leq \frac{1}{q} \wedge \frac{1}{p} \leq \frac{1}{2}\right] \text { or }\left[\frac{m-1}{m}+\frac{1}{m p}<\frac{1}{q} \wedge \frac{1}{2} \leq \frac{1}{p}\right] .
$$

- $(I I)$ : We know by $(I)$ that $\chi_{p, q_{m}}\left(\mathcal{P}\left({ }^{m} \mathbb{C}^{n}\right)\right) \sim 1$, for $\frac{1}{q_{m}}=\frac{1}{p}+\frac{m-1}{2 m}$. We now estimate $\chi_{p, \infty}\left(\mathcal{P}\left({ }^{m} \mathbb{C}^{n}\right)\right)$ for $0 \leq \frac{1}{p} \leq \frac{1}{2}$. Take $r=1$. By Proposition 2.4 and Theorem $2.1(C)$ we have

$$
B_{r, \infty}^{m}(n) \sim 1, A_{p, r}^{m}(n) \sim n^{m\left(\frac{1}{p}+\frac{1}{2}\right)-\frac{1}{2}} .
$$

Using Lemma 3.5

$$
\chi_{p, \infty}\left(\mathcal{P}\left({ }^{m} \mathbb{C}^{n}\right)\right) \ll n^{m\left(\frac{1}{p}+\frac{1}{2}\right)-\frac{1}{2}} .
$$


Take a polynomial $P \in \mathcal{P}\left({ }^{m} \mathbb{C}^{n}\right), P=\sum_{\mathbf{j} \in \mathcal{J}(m, n)} c_{\mathbf{j}} z_{\mathbf{j}}$ with $\|P\|_{\mathcal{P}\left(m \ell_{p}^{n}\right)}=1$ and take $\operatorname{signs}\left(\theta_{\mathbf{j}}\right)_{\mathbf{j} \in \mathcal{J}(m, n)}$. Therefore since

$$
\begin{aligned}
\left\|\sum_{\mathbf{j} \in \mathcal{J}(m, n)} \theta_{\mathbf{j}} c_{\mathbf{j}} z_{\mathbf{j}}\right\|_{\mathcal{P}\left({ }^{m} \ell_{q_{m}}^{n}\right)} & \leq \chi_{p, q_{m}}\left(\mathcal{P}\left({ }^{m} \mathbb{C}^{n}\right)\right) \sim 1 \\
\left\|\sum_{\mathbf{j} \in \mathcal{J}(m, n)} \theta_{\mathbf{j}} c_{\mathbf{j}} z_{\mathbf{j}}\right\|_{\mathcal{P}\left({ }^{m} \ell_{\infty}^{n}\right)} & \leq \chi_{p, \infty}\left(\mathcal{P}\left({ }^{m} \mathbb{C}^{n}\right)\right) \ll n^{m\left(\frac{1}{p}+\frac{1}{2}\right)-\frac{1}{2}}
\end{aligned}
$$

we have, by (6) and The Multilinear Interpolation Theorem (see [BL76, Section 4.4]) that for $\theta \in(0,1)$ and $\frac{1}{q}=\frac{\theta}{q_{m}}+\frac{1-\theta}{\infty}$,

$$
\left\|\sum_{\mathbf{j} \in \mathcal{J}(m, n)} \theta_{\mathbf{j}} c_{\mathbf{j}} z_{\mathbf{j}}\right\|_{\mathcal{P}\left(m \ell_{q}^{n}\right)} \ll n^{(1-\theta)\left[m\left(\frac{1}{p}+\frac{1}{2}\right)-\frac{1}{2}\right]}=n^{m\left(\frac{1}{p}-\frac{1}{q}+\frac{1}{2}\right)-\frac{1}{2}} .
$$

For the lower bound let $P(z)=\sum_{\alpha \in \Lambda(m, n)} \varepsilon_{\alpha} z^{\alpha}$ be a unimodular polynomial as in (4) with $p \geq 2$, then if $w=\left(\frac{1}{n^{1 / q}}, \ldots, \frac{1}{n^{1 / q}}\right) \in S_{\ell_{q}}$, we have

$$
\chi_{p, q}\left(\mathcal{P}\left({ }^{m} \mathbb{C}^{n}\right)\right) \gg \frac{\left\|\sum_{\alpha \in \Lambda(m, n)} z^{\alpha}\right\|_{\mathcal{P}\left(m \ell_{q}^{n}\right)}}{\left\|\sum_{\alpha \in \Lambda(m, n)} \varepsilon_{\alpha} z^{\alpha}\right\|_{\mathcal{P}\left({ }^{m} \ell_{p}^{n}\right)}} \gg \frac{\left|\sum_{\alpha \in \Lambda(m, n)} w^{\alpha}\right|}{n^{m\left(\frac{1}{2}-\frac{1}{p}\right)+\frac{1}{2}}} \gg \frac{n^{m\left(1-\frac{1}{q}\right)}}{n^{m\left(\frac{1}{2}-\frac{1}{p}\right)+\frac{1}{2}}}=n^{m\left(\frac{1}{p}-\frac{1}{q}+\frac{1}{2}\right)-\frac{1}{2}} .
$$

$\bullet(I I I)$ : For $\left[\frac{1}{2} \leq \frac{1}{p} \wedge \frac{1}{q} \leq \frac{1}{p}\right]$ let us take $\frac{1}{r}=1-\frac{1}{q}$. Note that $\frac{1}{r} \geq 1-\frac{1}{p}$. Then by Proposition 2.4 and Theorem $2.1(D)$,

$$
B_{r, q}^{m}(n) \sim 1, A_{p, r}^{m}(n) \sim n^{(m-1)\left(1-\frac{1}{q}\right)+\frac{1}{p}-\frac{1}{q}}
$$

and therefore

$$
\chi_{p, q}\left(\mathcal{P}\left({ }^{m} \mathbb{C}^{n}\right)\right) \ll n^{(m-1)\left(1-\frac{1}{q}\right)+\frac{1}{p}-\frac{1}{q}} .
$$

For the lower bound let $P(z)=\sum_{\alpha \in \Lambda(m, n)} \varepsilon_{\alpha} z^{\alpha}$ be a unimodular polynomial as in (44) with $1 \leq p \leq 2$, we have

$$
\chi_{p, q}\left(\mathcal{P}\left({ }^{m} \mathbb{C}^{n}\right)\right) \gg \frac{\left\|\sum_{\alpha \in \Lambda(m, n)} z^{\alpha}\right\|_{\mathcal{P}\left(m \ell_{q}^{n}\right)}}{\left\|\sum_{\alpha \in \Lambda(m, n)} \varepsilon_{\alpha} z^{\alpha}\right\|_{\mathcal{P}\left(m \ell_{p}^{n}\right)}} \gg \frac{n^{m\left(1-\frac{1}{q}\right)}}{n^{1-\frac{1}{p}}}=n^{(m-1)\left(1-\frac{1}{q}\right)+\frac{1}{p}-\frac{1}{q}} .
$$

$\bullet\left(I I I^{\prime}\right)$ : Let $\frac{1}{2}<\frac{1}{p}<1$. For every $\lambda>\frac{1}{p}$ we know by Lemma 3.8 that $\chi_{p, q_{m}}\left(\mathcal{P}\left({ }^{m} \mathbb{C}^{n}\right)\right) \ll$ $\log (n)^{m\left(\lambda+\frac{1}{q_{m}}\right)}$, where $\frac{1}{q_{m}}=1-\frac{1}{m}+\frac{1}{m p}$. On the other hand, by $(I I I)$ we know that $\chi_{p, p}\left(\mathcal{P}\left({ }^{m} \mathbb{C}^{n}\right)\right) \sim$ $n^{(m-1)\left(1-\frac{1}{p}\right)}$. 
As in $(I I)$ we will use the The Multilinear Interpolation Theorem: take a polynomial $P \in$ $\mathcal{P}\left({ }^{m} \mathbb{C}^{n}\right), P=\sum_{\mathbf{j} \in \mathcal{J}(m, n)} c_{\mathbf{j}} z_{\mathbf{j}}$ with $\|P\|_{\mathcal{P}\left({ }^{m} \ell_{p}^{n}\right)}=1$ and take signs $\left(\theta_{\mathbf{j}}\right)_{\mathbf{j} \in \mathcal{J}(m, n)}$. Therefore since

$$
\begin{aligned}
&\left\|\sum_{\mathbf{j} \in \mathcal{J}(m, n)} \theta_{\mathbf{j}} c_{\mathbf{j}} z_{\mathbf{j}}\right\|_{\mathcal{P}\left(m \ell_{q_{m}}^{n}\right)} \leq \chi_{p, q_{m}}\left(\mathcal{P}\left({ }^{m} \mathbb{C}^{n}\right)\right) \ll \log (n)^{m\left(\lambda+\frac{1}{q_{m}}\right)}, \\
&\left\|\sum_{\mathbf{j} \in \mathcal{J}(m, n)} \theta_{\mathbf{j}} c_{\mathbf{j}} z_{\mathbf{j}}\right\|_{\mathcal{P}\left({ }^{m} \ell_{p}^{n}\right)} \leq \chi_{p, p}\left(\mathcal{P}\left(^{m} \mathbb{C}^{n}\right)\right) \sim n^{(m-1)\left(1-\frac{1}{p}\right)},
\end{aligned}
$$

we have, by (6) and [BL76, Section 4.4], that for $\theta \in(0,1)$ and $\frac{1}{q}=\frac{\theta}{q_{m}}+\frac{1-\theta}{p}$,

$$
\begin{aligned}
\left\|\sum_{\mathbf{j} \in \mathcal{J}(m, n)} \theta_{\mathbf{j}} c_{\mathbf{j}} z_{\mathbf{j}}\right\|_{\mathcal{P}\left(m^{m} \ell_{q}^{n}\right)} & \ll \log (n)^{\theta m\left(\lambda+\frac{1}{q_{m}}\right)} n^{(1-\theta)\left[(m-1)\left(1-\frac{1}{p}\right)\right]} \\
& =\log (n)^{\theta m\left(\lambda+\frac{1}{q_{m}}\right)} n^{(m-1)\left(1-\frac{1}{q}\right)+\frac{1}{p}-\frac{1}{q}}
\end{aligned}
$$

with $\theta=\frac{m}{m-1} p^{\prime}\left(\frac{1}{q}-\frac{1}{p}\right)$. This concludes the upper bound for the region $\left(I I I^{\prime}\right):\left[1-\frac{1}{m}+\frac{1}{m p} \geq\right.$ $\left.\frac{1}{q} \geq \frac{1}{p} \wedge \frac{1}{2}<\frac{1}{p}<1\right]$.

The lower bound is exactly as in (15).

\section{Some applications to the multivariable von Neumann's inequality}

A classical inequality in operator theory, due to von Neumann [vN51, asserts that if $T$ is a linear contraction on a complex Hilbert space $\mathcal{H}$ (i.e., its operator norm is less than or equal to one) then

$$
\|p(T)\|_{\mathcal{L}(\mathcal{H})} \leq \sup \{|p(z)|: z \in \mathbb{C},|z| \leq 1\}
$$

for every polynomial $p$ in one (complex) variable.

Using dilation theory (see [SN74]), for polynomials in two commuting contractions, Ando And63 exhibited an analogue inequality. However Varopoulos Var74 showed that von Neumann's inequality cannot be extended for three or more commuting contractions.

It is an open problem of great interest in operator theory (see for example [Ble01, Pis01]) to determine whether there exists a constant $K(n)$ that adjusts von Neumann's inequality. More precisely, it is unknown whether or not there exists a constant $K(n)$ such that

$$
\left\|p\left(T_{1}, \ldots, T_{n}\right)\right\|_{\mathcal{L}(\mathcal{H})} \leq K(n) \sup \left\{\left|p\left(z_{1}, \ldots, z_{n}\right)\right|:\left|z_{i}\right| \leq 1\right\}
$$

for every polynomial $p$ in $n$ variables and every $n$-tuple $\left(T_{1}, \ldots, T_{n}\right)$ of commuting contractions in $\mathcal{L}(\mathcal{H})$. 
Dixon [Dix76] studied the multivariable von Neumann's inequality restricted to homogeneous polynomials and [MT79] studied some variations of this problem. One of them is to determine the asymptotic behavior of the best possible constant $c(n)=c_{m, p, q}(n)$ such that

$$
\left\|P\left(T_{1}, \ldots, T_{n}\right)\right\|_{\mathcal{L}(\mathcal{H})} \leq c(n)\|P\|_{\mathcal{P}\left(m \ell_{q}^{n}\right)}
$$

for every $n$-tuple $T_{1}, \ldots, T_{n}$ of commuting operators on a Hilbert space satisfying

$$
\sum_{i=1}^{n}\left\|T_{i}\right\|_{\mathcal{L}(\mathcal{H})}^{p} \leq 1
$$

and any $m$-homogeneous polynomial on $n$ variables, $P$. Some lower bounds were proven there and also some upper bounds were given for the case $p=q$. We will apply the results of Section 2 to show upper bounds for $c(n)$ for any $1 \leq p, q \leq \infty$.

Recall that given a bilinear form $a: X_{1} \times X_{2} \rightarrow \mathbb{C}$ its uniform norm is

$$
\|a\|_{B i l\left(X_{1} \times X_{2}\right)}:=\sup _{\left(x_{1}, x_{2}\right) \in B_{X_{1}} \times B_{X_{2}}}\left|a\left(x_{1}, x_{2}\right)\right| .
$$

We need the following lemma from [MT79] which is an easy consequence of the Grothendieck inequality. We prove it for the sake of completeness.

Lemma 4.1. For $i=1, \ldots, N, j=1, \ldots, M$ let $x_{i}, y_{j}$ be vectors in some Hilbert space $\mathcal{H}$ such the $\sum_{i=1}^{N}\left\|x_{i}\right\|_{\mathcal{H}}^{p} \leq 1$ and $\sum_{j=1}^{M}\left\|y_{j}\right\|_{\mathcal{H}}^{p} \leq 1$, and let $\left(a_{i, j}\right)_{i, j} \in \mathbb{C}^{N \times M}$. Then

$$
\left|\sum_{i, j} a_{i j}\left\langle x_{i}, y_{j}\right\rangle\right| \leq K_{G}\|a\|_{B i l\left(\ell_{p}^{N} \times \ell_{p}^{M}\right)},
$$

where $K_{G}$ denotes the Grothendieck constant and a is the bilinear form on $\mathbb{C}^{N} \times \mathbb{C}^{M}$ whose coefficients are the $a_{i j}$ 's.

Proof.

$$
\begin{aligned}
\left|\sum_{i, j} a_{i, j}\left\langle x_{i}, y_{j}\right\rangle\right| & \leq\left|\sum_{i, j} a_{i, j}\left\|x_{i}\right\|_{\mathcal{H}}\left\|y_{j}\right\|_{\mathcal{H}}\left\langle\frac{x_{i}}{\left\|x_{i}\right\|_{\mathcal{H}}}, \frac{y_{j}}{\left\|y_{j}\right\|_{\mathcal{H}}}\right\rangle\right| \\
& \leq K_{G} \sup \left\{\sum_{i, j} a_{i, j}\left\|x_{i}\right\|_{\mathcal{H}}\left\|y_{j}\right\|_{\mathcal{H}} \beta_{i} \gamma_{j}: \beta \in B_{\ell_{\infty}^{N}}, \gamma \in B_{\ell_{\infty}^{M}}\right\} \\
& \leq K_{G}\|a\|_{B i l\left(\ell_{p}^{N} \times \ell_{p}^{M}\right)} .
\end{aligned}
$$

Proposition 4.2. Let $T_{1}, \ldots, T_{n}$ be commuting operators on a Hilbert space $\mathcal{H}$ satisfying (17) and $P \in \mathcal{P}\left({ }^{m} \mathbb{C}^{n}\right)$. Then

$$
\left\|P\left(T_{1}, \ldots, T_{n}\right)\right\|_{\mathcal{L}(\mathcal{H})} \leq C A_{q, p^{\prime}}^{m-1}(n)\|P\|_{\mathcal{P}\left(m \ell_{q}^{n}\right)},
$$


where $C$ is constant independent of $n$.

Proof. Let $a_{\mathbf{i}}, \mathbf{i} \in \mathcal{M}(m, n)$ be the coefficients of the symmetric $m$-linear form $a$ associated to $P$, and let $x, y$ be unit vectors in $\mathcal{H}$. Note that we may also view $a$ as a bilinear form on $\mathbb{C}^{n^{m-1}} \times \mathbb{C}^{n}$, then by the previous lemma,

$$
\begin{aligned}
\left|\sum_{\mathbf{i} \in \mathcal{M}(m, n)} a_{\mathbf{i}}\left\langle T_{i_{1}} \ldots T_{i_{m}} x, y\right\rangle\right| & =\left|\sum_{(\mathbf{i}, j) \in \mathcal{M}(m-1, n) \times\{1, \ldots, n\}} a_{(\mathbf{i}, j)}\left\langle T_{i_{1}} \ldots T_{i_{m-1}} x, T_{j}^{*} y\right\rangle\right| \\
& \leq K_{G}\|a\|_{B i l\left(\ell_{p}^{n^{m-1}} \times \ell_{p}^{n}\right)}=K_{G} \sup _{\beta \in B_{\ell_{p}^{n}}}\left(\sum_{\mathbf{i} \in \mathcal{M}(m-1, n)}\left|\sum_{j=1}^{n} a_{(\mathbf{i}, j)} \beta_{j}\right|^{p^{\prime}}\right)^{1 / p^{\prime}} .
\end{aligned}
$$

Note that $\sum_{j=1}^{n} a_{(\mathbf{i}, j)} \beta_{j}$ are the coefficients of the $(m-1)$-linear form $a_{\beta}$ which is obtained by fixing one variable of $a$ at $\beta$, that is, $a_{\beta}\left(v_{1}, \ldots, v_{m-1}\right)=a\left(\beta, v_{1}, \ldots, v_{m-1}\right)$. Note also that the $p^{\prime}$-norm of the coefficients of $a_{\beta}$ is less than or equal to the $p^{\prime}$-norm of the coefficients of the associated polynomial $P_{\beta}$. Then, since $\left\|P_{\beta}\right\|_{\left.\mathcal{P}^{(m-1} \ell_{q}^{n}\right)} \leq e\|P\|_{\mathcal{P}\left({ }^{m} \ell_{q}^{n}\right)}$ (see for example Har72]), taking supremum over $x, y \in B_{\mathcal{H}}$ we have

$$
\left\|P\left(T_{1}, \ldots, T_{n}\right)\right\|_{\mathcal{L}(\mathcal{H})} \leq K_{G} \sup _{\beta \in B_{\ell_{p}^{n}}} A_{q, p^{\prime}}^{m-1}(n)\left\|P_{\beta}\right\|_{\mathcal{P}\left(m-1 \ell_{q}^{n}\right)} \leq K_{G} e A_{q, p^{\prime}}^{m-1}(n)\|P\|_{\mathcal{P}\left(m \ell_{q}^{n}\right)} .
$$

Remark 4.3. Taking $p=q$ and using Theorem 2.1] we recover the inequality proved in [MT79], that is, $c(n) \ll n^{\frac{m-2}{p^{\prime}}}$ if $p \leq 2$ and $c(n) \ll n^{\frac{m-2}{2}}$ if $p \geq 2$.

We also have the following corollary.

Corollary 4.4. Let $T_{1}, \ldots, T_{n}$ be commuting operators on a Hilbert space $\mathcal{H}$ satisfying (17). If $\left[\frac{1}{2} \leq \frac{1}{p^{\prime}} \leq \frac{m}{2(m-1)}-\frac{1}{q}\right]$ or $\left[\frac{1}{p^{\prime}} \leq \frac{1}{2} \wedge \frac{m-1}{q} \leq 1-\frac{1}{p^{\prime}}\right]$, we have that

$$
\left\|P\left(T_{1}, \ldots, T_{n}\right)\right\|_{\mathcal{L}(\mathcal{H})} \leq D\|P\|_{\mathcal{P}\left(\ell_{q}^{n}\right)},
$$

for every m-homogeneous polynomial $P$, where $D$ is constant independent of $n$.

Another variant studied in [MT79] is to determine the best possible constant $d(n)=d_{m, p, q}(n)$ such that

$$
\left\|P\left(T_{1}, \ldots, T_{n}\right)\right\|_{\mathcal{L}(\mathcal{H})} \leq d(n)\|P\|_{\mathcal{P}\left(\ell_{q}^{n}\right)},
$$


for every $m$-homogeneous polynomial in $n$ variables, $P$, and every $n$-tuple $T_{1}, \ldots, T_{n}$ of commuting operators on a Hilbert space $\mathcal{H}$ satisfying

$$
\left(\sum_{i=1}^{n}\left|\left\langle T_{i} x, y\right\rangle\right|^{p}\right)^{1 / p} \leq\|x\|_{\mathcal{H}}\|y\|_{\mathcal{H}},
$$

for any vectors $x, y \in \mathcal{H}$. Note that (18) is equivalent to $\left\|\sum_{i=1}^{n} T_{i} \beta_{i}\right\|_{\mathcal{L}(\mathcal{H})} \leq\|\beta\|_{p^{\prime}}$, for every $\beta \in \mathbb{C}^{n}$.

Lemma 4.5. Let $T_{1}, \ldots, T_{n} \in \mathcal{L}(\mathcal{H})$ be operators satisfying (18), and let $x, y \in \mathcal{H}$. Then if $Q$ is the $m$-homogeneous polynomial in $n$ variables defined by

$$
Q(z)=\sum_{\mathbf{i} \in \mathcal{M}(m, n)}\left\langle T_{i_{1}} \ldots T_{i_{m}} x, y\right\rangle z_{i_{1}} \ldots z_{i_{m}},
$$

we have $\|Q\|_{\mathcal{P}\left(m \ell_{p^{\prime}}^{n}\right)} \leq\|x\|_{\mathcal{H}}\|y\|_{\mathcal{H}}$.

Proof.

$$
\begin{aligned}
& \|Q\|_{\mathcal{P}\left(m \ell_{p^{\prime}}^{n}\right)}=\sup _{z \in B_{\ell_{p^{\prime}}^{n}}}\left|\sum_{\mathbf{i} \in \mathcal{M}(m, n)}\left\langle T_{i_{1}} \ldots T_{i_{m}} x, y\right\rangle z_{i_{1}} \ldots z_{i_{m}}\right|=\sup _{z \in B_{\ell_{p^{\prime}}^{n}}}\left|\left\langle\sum_{\mathbf{i} \in \mathcal{M}(m, n)} T_{i_{1}} \ldots T_{i_{m}} z_{i_{1}} \ldots z_{i_{m}} x, y\right\rangle\right| \\
& \leq \sup _{z \in B_{\ell^{n}}}\left|\left\langle\left(\sum_{l=1}^{n} z_{l} T_{l}\right)^{m} x, y\right\rangle\right| \leq \sup _{z \in B_{\ell^{n}}{ }^{\prime}}\left\|\sum_{l=1}^{n} z_{l} T_{l}\right\|^{m}\|x\|_{\mathcal{H}}\|y\|_{\mathcal{H}} \leq\|x\|_{\mathcal{H}}\|y\|_{\mathcal{H}} .
\end{aligned}
$$

Proposition 4.6. Let $T_{1}, \ldots, T_{n}$ be commuting operators on a Hilbert space $\mathcal{H}$ satisfying (18) and $P \in \mathcal{P}\left({ }^{m} \mathbb{C}^{n}\right)$. Then

$$
\left\|P\left(T_{1}, \ldots, T_{n}\right)\right\|_{\mathcal{L}(\mathcal{H})} \leq A_{q, r}^{m}(n) A_{p^{\prime}, r^{\prime}}^{m}(n)\|P\|_{\mathcal{P}\left(\ell_{q}^{m}\right)}
$$

Proof. Let $a_{\mathbf{i}}$ be the coefficients of the symmetric $m$-linear form $a$ associated to $P$ and $x, y$ unit vectors in $\mathcal{H}$. Then by the previous lemma and the fact that the $r$-norm of the coefficients of $a$ is less than or equal to the $r$-norm of the coefficients of the associated polynomial $P$, we have

$$
\begin{aligned}
\left|\sum_{\mathbf{i} \in \mathcal{M}(m, n)} a_{\mathbf{i}}\left\langle T_{i_{1}} \ldots T_{i_{m}} x, y\right\rangle\right| & \leq\left(\sum_{\mathbf{i} \in \mathcal{M}(m, n)}\left|a_{\mathbf{i}}\right|^{r}\right)^{1 / r}\left(\sum_{\mathbf{i} \in \mathcal{M}(m, n)}\left|\left\langle T_{i_{1}} \ldots T_{i_{m}} x, y\right\rangle\right|^{r^{\prime}}\right)^{1 / r^{\prime}} \\
& \leq A_{q, r}^{m}(n)\|P\|_{\mathcal{P}\left(m \ell_{q}^{n}\right)} A_{p^{\prime}, r^{\prime}}^{m}(n) .
\end{aligned}
$$


Remark 4.7. Taking $p=q=r^{\prime}$ and using Theorem 2.1 we recover the inequality proved in [MT79, Proposition 20], that is $d(n) \ll n^{(m-1)\left(\frac{1}{p^{\prime}}+\frac{1}{2}\right)}$ if $p \leq 2$ and $d(n) \ll n^{(m-1)\left(\frac{1}{p}+\frac{1}{2}\right)}$ if $p \geq 2$. Note also that, in the last proposition, we have bounds that do not depend on $n$ for some combinations of $p$ and $q$, e.g. for $(p, q)=(1, \infty)$.

\section{REFERENCES}

[ABPSS13] Nacib Albuquerque, Frédéric Bayart, Daniel Pellegrino, and Juan B Seoane-Sepúlveda. Optimal Hardy-Littlewood type inequalities for polynomials and multilinear operators. arXiv preprint arXiv:1311.3177, 2013.

[And63] Tsuyoshi Andô. On a pair of commutative contractions. Acta Sci. Math. (Szeged), 24:88-90, 1963.

[AP15] Gustavo Araujo and Daniel Pellegrino. Optimal Hardy-Littlewood type inequalities for $m$-linear forms on $\ell_{p}$ spaces with $1 \leq p \leq m$. arXiv preprint arXiv:1502.01522, 2015.

[Bay02] Frédéric Bayart. Hardy spaces of Dirichlet series and their composition operators. Monatshefte für Mathematik, 136(3):203-236, 2002.

[Bay12] Frédéric Bayart. Maximum modulus of random polynomials. The Quarterly Journal of Mathematics, 63(1):21-39, 2012.

[BPR18] Frédéric Bayart, Daniel Pellegrino, and Pilar Rueda. On coincidence results for summing multilinear operators: interpolation, $\ell_{1}$-spaces and cotype. arXiv preprint arXiv:1805.12500, 2018.

[BBEM90] Bernard Beauzamy, Enrico Bombieri, Per Enflo, and Hugh L Montgomery. Products of polynomials in many variables. Journal of Number Theory, 36(2):219-245, 1990.

[BCQ06] Ramachandran Balasubramanian, Bruno Calado, and Hervé Queffélec. The Bohr inequality for ordinary Dirichlet series. Studia Math, 175(3):285-304, 2006.

$\left[\mathrm{BDF}^{+} 14\right] \quad$ Frédéric Bayart, Andreas Defant, Leonhard Frerick, Manuel Maestre, and Pablo Sevilla-Peris. Multipliers of dirichlet series and monomial series expansions of holomorphic functions in infinitely many variables. arXiv preprint arXiv:1405.7205, 2014.

[BDS15] Frédéric Bayart, Andreas Defant, and Sunke Schlütters. Monomial convergence for holomorphic functions on $\ell_{r}$. Preprint, 2015.

[BH31] Henri Frédéric Bohnenblust and Einar Hille. On the absolute convergence of Dirichlet series. Annals of Mathematics, pages 600-622, 1931.

[BL76] Jöran Bergh and Jörgen Löfström. Interpolation spaces. An introduction. Springer-Verlag, Berlin, 1976. Grundlehren der Mathematischen Wissenschaften, No. 223.

[Ble01] Ron Blei. Analysis in integer and fractional dimensions, volume 71 of Cambridge Studies in Advanced Mathematics. Cambridge University Press, Cambridge, 2001.

[BMP10] Geraldo Botelho, Carsten Michels, and Daniel Pellegrino. Complex interpolation and summability properties of multilinear operators. Revista matemática complutense, 23(1):139-161, 2010.

[Boa00] Harold P Boas. Majorant series. J. Korean Math. Soc, 37(2):321-337, 2000.

[BPSS14] Frédéric Bayart, Daniel Pellegrino, and Juan B Seoane-Sepúlveda. The Bohr radius of the ndimensional polydisk is equivalent to $\sqrt{\frac{(\log n)}{n}}$. Advances in Mathematics, 264:726-746, 2014.

[CD07] Daniel Carando and Verónica Dimant. Extension of polynomials and John's theorem for symmetric tensor products. Proceedings of the American Mathematical Society, 135(6):1769-1773, 2007. 
$\left[\mathrm{CDG}^{+} 14\right]$ Daniel Carando, Andreas Defant, Domingo García, Manuel Maestre, and Pablo Sevilla-Peris. The Dirichlet-Bohr radius. arXiv preprint arXiv:1412.5947, 2014.

[CG11] Daniel Carando and Daniel Galicer. Unconditionality in tensor products and ideals of polynomials, multilinear forms and operators. The Quarterly Journal of Mathematics, 62(4):845-869, 2011.

[CKP99] Fernando Cobos, Thomas Kühn, and Jaak Peetre. On $g_{p}$-classes of trilinear forms. Journal of the London Mathematical Society, 59(3):1003-1022, 1999.

[DDGM01] Andreas Defant, Juan Carlos Díaz, Domingo García, and Manuel Maestre. Unconditional basis and Gordon-Lewis constants for spaces of polynomials. Journal of Functional Analysis, 181(1):119-145, 2001.

[DF11] Andreas Defant and Leonhard Frerick. The Bohr radius of the unit ball of $\ell_{p}^{n}$. Journal für die reine und angewandte Mathematik (Crelle's Journal), 2011(660):131-147, 2011.

$\left[\mathrm{DFOC}^{+} 11\right]$ Andreas Defant, Leonhard Frerick, Joaquim Ortega-Cerda, Myriam Ounaïes, and Kristian Seip. The Bohnenblust-Hille inequality for homogeneous polynomials is hypercontractive. Annals of mathematics, 174(1):485-497, 2011.

[DGM03] Andreas Defant, Domingo García, and Manuel Maestre. Bohr's power series theorem and local Banach space theory-to the memory of our friend Klaus Floret. Journal fur die reine und angewandte Mathematik, 557:173-197, 2003.

[DGM04] Andreas Defant, Domingo Garcia, and Manuel Maestre. Maximum moduli of unimodular polynomials. Journal of the Korean Mathematical Society, 41(1):209-229, 2004.

[Din99] Seán Dineen. Complex analysis on infinite dimensional spaces. Springer Monographs in Mathematics. London: Springer, 1999.

[Dix76] Peter G Dixon. The von Neumann inequality for polynomials of degree greater than two. Journal of the London Mathematical Society, 2(2):369-375, 1976.

[dLB08] Regis de La Breteche. Sur l'ordre de grandeur des polynômes de Dirichlet. Acta Arithmetica, 134:141148, 2008.

[DM00] Andreas Defant and Carsten Michels. A complex interpolation formula for tensor products of vectorvalued banach function spaces. Archiv der Mathematik, 74(6):441-451, 2000.

[DM03] Andreas Defant and Mieczyslaw Mastylo. On interpolation of tensor products of Banach spaces. Revista de la Real Academia de Ciencias Exactas, Físicas y Naturales. Serie A: Matemáticas (RACSAM), 97(2):209, 2003.

[DM15] Andreas Defant and Mieczysław Mastyło. $L^{p}$-norms and Mahler's measure of polynomials on the n-dimensional torus. Constructive Approximation, pages 1-15, 2015.

[DMP09] Andreas Defant, Manuel Maestre, and Christopher Prengel. Domains of convergence for monomial expansions of holomorphic functions in infinitely many variables. Journal für die reine und angewandte Mathematik (Crelles Journal), 2009(634):13-49, 2009.

[DSP13] Verónica Dimant and Pablo Sevilla-Peris. Summation of coefficients of polynomials on $\ell_{p}$ spaces. arXiv preprint arXiv:1309.6063, 2013.

[Fen03] Bao Qi Feng. Equivalence constants for certain matrix norms. Linear algebra and its applications, 374:247-253, 2003.

[FT07] Bao Qi Feng and Andrew Tonge. Equivalence constants for certain matrix norms II. Linear Algebra and Its Applications, 2(420):388-399, 2007. 
[GMSP15] Daniel Galicer, Santiago Muro, and Pablo Sevilla-Peris. Asymptotic estimates on the von neumann inequality for homogeneous polynomials. Journal für die reine und angewandte Mathematik (Crelle's Journal), accepted. (arXiv: 1504.05547), 2015.

[Gol87] Moshe Goldberg. Equivalence constants for $l_{p}$ norms of matrices. Linear and Multilinear Algebra, 21(2):173-179, 1987.

[Har72] Lawrence A Harris. Bounds on the derivatives of holomorphic functions of vectors. In Proc. Colloq. Analysis, Rio de Janeiro, volume 145, page 163, 1972.

[HL34] Godfrey Harold Hardy and John Edensor Littlewood. Bilinear forms bounded in space [p, q]. The Quarterly Journal of Mathematics, (1):241-254, 1934.

[Kah93] Jean-Pierre Kahane. Some random series of functions, volume 5. Cambridge University Press, 1993.

[Kou91] Omran Kouba. On the interpolation of injective or projective tensor products of banach spaces. Journal of Functional Analysis, 96(1):38-61, 1991.

[KQ01] Sergei V Konyagin and Hervé Queffélec. The translation 1/2 in the theory of Dirichlet series. Real Analysis Exchange, 27(1):155-176, 2001.

[Lit30] John E Littlewood. On bounded bilinear forms in an infinite number of variables. The Quarterly Journal of Mathematics, 1:164-174, 1930.

[Mon12] Ashley Montanaro. Some applications of hypercontractive inequalities in quantum information theory. Journal of Mathematical Physics, 53(12):122206, 2012.

[MT79] Anna Maria Mantero and Andrew Tonge. Banach algebras and von Neumann's inequality. Proceedings of the London Mathematical Society, 3(2):309-334, 1979.

[MT80] Anna Mantero and Andrew Tonge. The Schur multiplication in tensor algebras. Studia Mathematica, 68(1):1-24, 1980.

[Pis78] Gilles Pisier. Some results on Banach spaces without local unconditional structure. Compositio Mathematica, 37(1):3-19, 1978.

[Pis01] Gilles Pisier. Similarity problems and completely bounded maps, volume 1618 of Lecture Notes in Mathematics. Springer-Verlag, Berlin, expanded edition, 2001. Includes the solution to "The Halmos problem".

[PP81] T Praciano-Pereira. On bounded multilinear forms on a class of $\ell_{p}$ spaces. Journal of Mathematical Analysis and Applications, 81(2):561-568, 1981.

[Que95] Hervé Queffélec. H. Bohr's vision of ordinary Dirichlet series; old and new results. J. Anal, 3:43-60, 1995.

[Röd85] Vojtech Rödl. On a packing and covering problem. European J. Combin., 6(1):69-78, 1985.

[Sch78] Carsten Schütt. Unconditionality in tensor products. Israel Journal of Mathematics, 31(3):209-216, 1978.

[SN74] Béla Sz.-Nagy. Unitary dilations of Hilbert space operators and related topics. American Mathematical Society, Providence, R.I., 1974. Expository Lectures from the CBMS Regional Conference held at the University of New Hampshire, Durham, N.H., June 7-11, 1971, Conference Board of the Mathematical Sciences Regional Conference Series in Mathematics, No. 19.

[Sza81] Stanislaw Jerzy Szarek. A note on the paper of Schütt "Unconditionality in tensor products". In Colloquium Mathematicae, volume 45, pages 273-276. Institute of Mathematics Polish Academy of Sciences, 1981. 
[Ton00] Andrew Tonge. Equivalence constants for matrix norms: a problem of Goldberg. Linear Algebra and its Applications, 306(1):1-13, 2000.

[Var74] Nicholas Th. Varopoulos. On an inequality of von Neumann and an application of the metric theory of tensor products to operators theory. J. Functional Analysis, 16:83-100, 1974.

[vN51] Johann von Neumann. Eine Spektraltheorie für allgemeine Operatoren eines unitären Raumes. Math. Nachr., 4:258-281, 1951.

[Wei80] Fred B Weissler. Logarithmic Sobolev inequalities and hypercontractive estimates on the circle. Journal of Functional Analysis, 37(2):218-234, 1980.

Daniel Galicer. Departamento de Matemática - Pab i, Facultad de Cs. Exactas y Naturales, Universidad de Buenos Aires (1428) Buenos Aires, Argentina and imas-COnicet

E-mail address: dgalicer@dm.uba.ar

Martín Mansilla. Departamento de Matemática - Pab i, Facultad de Cs. Exactas y Naturales, Universidad de Buenos Aires (1428) Buenos Aires, Argentina and imas-COniCET

E-mail address: mmansilla@dm.uba.ar

Santiago Muro. Departamento de Matemática - Pab I, Facultad de Cs. Exactas y Naturales, Universidad de Buenos Aires, (1428) Buenos Aires, Argentina and CifASis-ConiCET

E-mail address: muro@cifasis-conicet.gov.ar 